\title{
Reduced expression of microRNA-139-5p in hepatocellular carcinoma results in a poor outcome: An exploration the roles of microRNA-139-5p in tumorigenesis, advancement and prognosis at the molecular biological level using an integrated meta-analysis and bioinformatic investigation
}

\author{
HUI QIN ${ }^{1}$, DONG-YUE WEN ${ }^{1}$, QIAO QUE ${ }^{1}$, CHUAN-YANG ZHOU $^{1}$, XIAO-DONG WANG $^{1}$, \\ YU-TING PENG ${ }^{1}$, YUN HE ${ }^{1}$, HONG YANG ${ }^{1}$ and BO-MING LIAO ${ }^{2}$ \\ Departments of ${ }^{1}$ Medical Ultrasonics and ${ }^{2}$ Internal Medicine, The First Affiliated Hospital of Guangxi Medical University, \\ Nanning, Guangxi Zhuang Autonomous Region 530021, P.R. China
}

Received May 10, 2019; Accepted September 27, 2019

DOI: $10.3892 / 01.2019 .11031$

\begin{abstract}
Hepatocellular carcinoma (HCC) is generally considered one of the most common gastrointestinal malignant tumors, characterized by high invasiveness and metastatic rate, as well as insidious onset. A relationship between carcinogenicity and aberrant microRNA-139-5p (miR-139-5p) expression has been identified in multiple tumors while the specific molecular mechanisms of miR-139-5p in HCC have not yet been thoroughly elucidated. A meta-analysis of available data from The Cancer Genome Atlas (TCGA), Gene Expression Omnibus, ArrayExpress and Oncomine databases, as well as the published literature, was comprehensively conducted with the aim of examining the impact of miR-139-5p expression on HCC. Additionally, predicted downstream target genes were confirmed using a series of bioinformatics tools. Moreover, a correlative biological analysis was performed to ascertain the precise function of miR-139-5p in HCC. The results revealed that the expression of miR-139-5p was noticeably lower in HCC compared with non-tumor liver tissues according to the pooled standard mean difference, which was -0.84 [95\% confidence interval $(\mathrm{CI}):-1.36$ to $-0.32 ; \mathrm{P}<0.001]$. Furthermore, associations were detected between miR-139-5p expression and certain clinicopathological characteristics of TCGA samples, including tumor grade, pathological stage and $\mathrm{T}$ stage. Moreover, the pooled hazard ratio (HR) for overall survival $(\mathrm{HR}=1.37 ; 95 \% \mathrm{CI}: 1.07-1.76 ; \mathrm{P}=0.001)$ indicated
\end{abstract}

Correspondence to: Professor Bo-Ming Liao, Department of Internal Medicine, The First Affiliated Hospital of Guangxi Medical University, 6 Shuangyong Road, Nanning, Guangxi Zhuang Autonomous Region 530021, P.R. China

E-mail:2369204378@qq.com

Key words: hepatocellular carcinoma,progressive, clinicopathological features, bioinformatics analysis, microRNA-139-5p that decreased miR-139-5p expression was a risk factor for adverse outcomes. Additionally, 382 intersecting genes regulated by miR-139-5p were obtained and assembled in signaling pathways, including 'transcription factor activity, sequence-specific DNA binding', 'pathways in cancer' and 'Ras signaling pathway'. Notably, four targeted genes that were focused in 'pathways in cancer' were identified as hub genes and immunohistochemical staining of the proteins encoded by these four hub genes in liver tissues, explored using the Human Protein Atlas database, confirmed their expression patterns in HCC and normal liver tissues Findings of the present study suggest that reduced miR-139-5p expression is capable of accelerating tumor progression and is associated with a poor clinical outcome by modulating the expression of downstream target genes involved in tumor-associated signaling pathways.

\section{Introduction}

The increasing incidence and mortality rates of cancer in humans worldwide have exerted serious effects on the health and quality of life of affected individuals, particularly in developed countries $(1,2)$. Hepatocellular carcinoma (HCC), a very common malignant gastrointestinal tumor that occurs in geographically heterogeneous regions, in particular Eastern Asia and sub-Saharan Africa, where medical service is relatively limited, accounts for $75-85 \%$ of primary liver cancer cases. The major risk factors, including chronic hepatitis $\mathrm{B}$ and hepatitis $\mathrm{C}$, excessive intake of alcohol, aflatoxin exposure and metabolic liver disease, have increased the threat of HCC worldwide (2). HCC has been revealed to have sex-specific differences, as the prevalence and mortality rates are 2-3 times higher among men than women $(3,4)$. This predisposition may be attributed to the frequent development of chronic hepatitis $\mathrm{B}$ virus infection, alcoholism, fatty liver disease and hepatitis $C$ virus infection in men (5-7). Although substantial advances have been made in the therapeutic field of $\mathrm{HCC}$, frequent recurrence, advanced diagnosis and metastasis remain challenging issues to overcome and are preventative factors in obtaining a 
good prognosis $(8,9)$. Thus, a useful marker for estimating the diagnosis and curative outcome of HCC is essential.

Mature microRNAs (miRNAs), commonly recognized as a major family of endogenous and small non-coding RNAs with a length of $\sim 22$ nucleotides, bind to the 3'-untranslated region (UTR) of corresponding target messenger RNAs (mRNAs), resulting in the inhibition of protein translation or degradation of mRNAs (10-12). Importantly, it is generally acknowledged that miRNAs serve important roles in biological processes, including cell proliferation, expansion, differentiation, infiltration and apoptosis, by regulating the expression of related genes in tissues or blood plasma (13-15). Moreover, miRNA dysregulation is an important factor contributing to tumorigenesis and progression, furthermore, certain specific miRNAs, such as miR-92a-2, miR-133b, miR-34a and miR-96, can act as either oncogenes or tumor suppressors (16-18).

miR-139-5p, one of the most common subtypes of miR-139, which is located on chromosome 11q13.4, has become established as a critical focus of studies examining the initiation and progression of diverse cancers, including oral squamous carcinoma (19), colorectal cancer $(20,21)$, bladder cancer (22), endometrial cancer (23) and HCC (24-27). Wang et al (27) reported that miR-139-5p inhibits HCC development, however, the study included a limited number of HCC specimens. Moreover, a number of studies have reported a close association between differential miR-139-5p expression and the prognosis of HCC $(25,26,28,29)$. Nevertheless, literature reporting the precise molecular mechanisms and available published studies analyzing miR-139-5p regulation in HCC are inconclusive, thus additional comprehensive studies are required.

The current meta-analysis was performed by integrating miRNA sequencing and microarray data, and comprehensively analyzing published articles that mentioned a relationship between miR-139-5p regulation and clinical data from human HCC samples. In addition, a bioinformatics analysis was conducted in order to confirm the characteristics of miR-139-5p and its targeted genes in HCC pathology- and development-related mechanisms. The present meta-analysis may provide further suggestions regarding the development of novel molecular therapeutic strategies for patients with HCC.

\section{Materials and methods}

Data mining and literature search. Microarray profiles associated with miRNA expression in HCC were searched and downloaded from the Gene Expression Omnibus (GEO) (30), The Cancer Genome Atlas (TCGA) (31), ArrayExpress (32) and Oncomine (33) databases. The related records were updated on March 28, 2019 and the following search terms were used: (malignan* OR cancer OR tumor OR tumour OR neoplas* OR carcinoma) AND (hepatocellular OR liver OR hepatic OR HCC). Initially, studies were chosen based on the following inclusion criteria: i) Human HCC confirmed by pathological analysis and ii) information about miR-139-5p regulation in HCC and nontumorous samples.

For the published literature search, scientific databases, including PubMed (https://www.ncbi.nlm.nih.gov/pubmed/), ScienceDirect (https://www.sciencedirect.com/), Web of Science (http://wokinfo.com/), Wang Fang (http://www.wanfangdata. com.cn/index.html), ChongQing VIP (http://cstj.cqvip.com/) and the China National Knowledge Infrastructure (https://www. cnki.net/), were comprehensively searched to determine the most relevant studies published up to March 28, 2019. The key search terms were: (miR-139-5p OR miRNA-139-5p OR microRNA139-5p OR miR139-5p OR miRNA139-5p OR miR 139-5p OR miRNA 139-5p OR microRNA 139-5p OR microRNA-139-5p) AND (malignan* OR cancer OR tumor OR tumour OR neoplas* OR carcinoma) AND (hepatocellular OR liver OR hepatic OR HCC). The inclusion criteria regarding the published studies were: i) HCC confirmed by an operation and pathological analysis; ii) information on the miR-139-5p expression levels (high vs. low) and relative clinicopathological data, including odds ratios (ORs) with $95 \%$ confidence intervals (CIs) of some clinicopathological parameters in $\mathrm{HCC}$; and iii) direct clinical prognostic information, including overall survival (OS), recurrence-free survival (RFS) or disease-free survival (DFS) with 95\% CIs. Furthermore, when the Kaplan-Meier curves were drawn clearly, we could artificially extract and calculate the effect sizes, including OS, RFS, DFS with corresponding 95\% CIs statistically.

The following exclusion criteria were applied: i) Lack of information on miR-139-5p expression in patients with HCC; ii) the full study was not published in English or Chinese; iii) reported data were insufficient for the calculation of clinical pathological parameters and prognostic indicators; and iv) the articles were reviews, conference abstracts or case reports.

Data extraction. The following information was considered for microarray data selection: Dataset name, case number $(\mathrm{N})$, mean (M) and standard deviation (SD) of corresponding datasets among HCC and nontumorous tissues, as well as area under the curve (AUC) values. Available parameters from the TCGA clinicopathological analysis included: Age, sex, race, neoplasm status, tumor grade, pathological stage, tumor-node-metastasis (TNM) stage, lymph node metastasis and metastasis status.

The following data were extracted from the scientific publications: First author, year published, country, sample sizes, methods, sample sources, follow-up time, outcomes and hazard ratios (HRs) with corresponding 95\% CIs for OS, RFS or DFS. Kaplan-Meier curves were generated using the officially recommended method by Tierney et al (34).

Statistical analysis. Statistical analyses were performed using Stata (version 14.0; StataCorp LP), SPSS software (version 24.0; SPSS, IBM Corp.) and GraphPad Prism software (version 9; GraphPad Software, Inc.). Initially, microarray data were separated into two groups, namely the HCC tissue group and the nontumorous liver tissue group. miR-139-5p expression levels were calculated from each included dataset, the $\mathrm{N}$ and $\mathrm{M} \pm \mathrm{SD}$ of the two groups were then recorded and compared using Student's t-test. More than two groups in the TCGA clinical parameter calculation were analyzed using one-way ANOVA. In addition, scatter plots presenting miR-139-5p expression levels were generated using GraphPad Prism software, and the receiver operating characteristic (ROC) curve analysis of each dataset was performed using SPSS software. Meta-DiSc (version 1.4; Clinical Biostatistics Unit Miscellaneous Shareware), which is capable of testing the precision of meta-analyses, was utilized to assess diagnostic efficiency, including the summary receiver operating 
characteristic (sROC) curve, positive likelihood ratio (PLR), negative likelihood ratio (NLR), and diagnostic sensitivity and specificity. Moreover, the merged standard mean differences (SMDs) and 95\% CIs for all appropriate datasets were assessed using Stata 14.0 software to analyze miR-139-5p expression levels in patients with HCC. The merged SMD was $<0$ and the $95 \% \mathrm{CI}$ did not include 0 , indicating a relatively low expression level of miR-139-5p in HCC tissues. Furthermore, Kaplan-Meier survival curves were manually extracted from published studies via Engauge Digitizer software (version 4.1; http://markummitchell.github.io/engauge-digitizer/), and outcomes were recorded as HRs and 95\% CIs. These values were ultimately merged using Stata 14.0 software aimed at confirming the prognostic effect of miR-139-5p. The HR was considered significant if $\mathrm{P}<0.05$ and the $95 \% \mathrm{CI}$ did not exceed 1 ; otherwise, no significant effect of miR-139-5p regulation was observed in patients with HCC. Heterogeneity analysis was evaluated by the Cochrane Q and inconsistency index $\left(\mathrm{I}^{2}\right)$ tests (35). A random-effects model was employed if clear heterogeneity was indicated with $\mathrm{I}^{2}>50 \%$ or $\mathrm{P}<0.05$; if not, a fixed-effects model was selected. Additionally, publication bias was detected by Begg's funnel test, and influence analysis was performed to determine whether the studies or datasets included in the present study exerted excessive effects on pooled outcomes by independently removing each study.

Bioinformatics analysis. Twelve online tools, namely miRWalk3.0 (http://mirwalk.umm.uni-heidelberg. de/), MiRTarBase (http://mirtarbase.mbc.nctu.edu. tw/php/index.php), TarBase6.0 (http://www.microrna. $\mathrm{gr} / \mathrm{tarbase}$ ), TargetMiner (http://www.mybiosoftware. com/targetminer-microrna-target-prediction.html), polymiRTS3.0 (http://compbio.uthsc.edu/miRSNP), RNA22 (https://cm.jefferson.edu/rna22/), microRNA.org (http://www.microrna.org/), PITA (https://genie.weizmann. ac.il/pubs/mir07/mir07_data.html), mirRNAMAP2.0 (http://mirnamap.mbc.nctu.edu.tw/), TargetScan (http://www.targetscan.org/vert_71/), miRDB (http://mirdb. org/) and PicTar-vert (http://dorina.mdc-berlin.de) (36-47), were employed to comprehensively investigate the impact of miR-139-5p on biological processes. Genes were investigated to predict possible target genes and differentially expressed genes (DEGs) in HCC tissues were downloaded from TCGA. Next, a Venn diagram (http://bioinfogp.cnb.csic.es/tools/venny/) was generated to obtain intersecting DEGs as final target genes from the aforementioned DEGs and predicted genes. Furthermore, the final target genes were subjected to Gene Ontology (GO) enrichment, Kyoto Encyclopedia of Genes and Genomes (KEGG) and Panther pathway analyses using the Database for Annotation, Visualization and Integrated Discovery (DAVID; version 6.8; http://david.ncifcrf.gov) (48). $\mathrm{P}<0.05$ was considered to indicate a statistically significant difference in all pathways. Moreover, images of correlation networks were presented in a bubble diagram that was constructed using the ImageGP online website (http://www. ehbio.com/ImageGP/). The Search Tool for the Retrieval of Interacting Genes (STRING; http://string-db.org; version 11.0) was employed to explore the protein-protein interaction (PPI) network and identify the top downstream target genes with the greatest number of connections of each node that were most likely regulated by miR-139-5p in HCC $(49,50)$. Additionally, Gene Expression Profiling Interactive Analysis (GEPIA; http://gepia.cancer-pku.cn/; version 1.0) (51) was used to determine the expression and relative prognostic roles of downstream target genes in the development of HCC. Intersecting DEGs were identified as hub genes from the top downstream genes and the target genes that were prominently enriched in the first pathway, which included the most statistically significant differences of KEGG. Additionally, the Human Protein Atlas database (52) and CBio Cancer Genomics Portal (53) were searched to determine the deregulation and mutations of hub genes. The expression patterns of hub genes were validated using immunohistochemical (IHC) staining images of pathological sections obtained from the Human Protein Atlas, and comparing normal and HCC tissue samples. The correlation between hub gene and miR-139-5p expression in TCGA data was evaluated by the calculation of Pearson's correlation coefficients using SPSS software.

\section{Results}

Characteristics of the included studies, miRNA-sequencing (miRNA-seq, and microarray data. In the literature search performed using the aforementioned retrieval strategy, 205 studies were preliminarily obtained. After thorough examination of all the studies, 201 studies were excluded due to 36 articles being duplicated, and no available clinical information being explicitly provided in 165 articles. Consequently, only four studies that provided associations with the survival of 367 patients could be included $(25,28,29,42)$. Three studies presented survival curve data that allowed OS calculation, and one directly provided accurate OS data. However, only one study separately $(28,29)$ described DFS and RFS, which were not merged to confirm the significance of these two effect sizes. Details of the included terms are presented in Table I, and a schematic of the literature and microarray data screening processes is shown in Fig. 1.

The miRNA-seq and microarray data were initially retrieved from 90,880 datasets, and after careful examination and verification of the data, 18 datasets were identified to provide miR-139-5p expression data regarding HCC and nontumor liver tissues that conformed to the inclusion criteria of the present study. These 18 datasets were retrieved from the TCGA, GEO, ArrayExpress and Oncomine databases. Specifically, 14 datasets were obtained from GEO and Oncomine, including GSE12717, GSE21279, GSE21362, GSE36915, GSE39678, GSE40744, GSE41874, GSE50013, GSE54751, GSE64632, GSE67882, GSE69580, GSE98269 and GSE115016. Three datasets were retrieved from the ArrayExpress database: E-MTAB-511, E-MTAB-3347 and E-MTAB-4809. The remaining TCGA dataset containing available data of miR-139-5p expression profile was downloaded from TCGA miRNA-seq profiles. Detailed information is presented in Table II.

Analysis of miRNA-seq and microarray data acquired from public databases and the scientific literature

miR-139-5p expression data in HCC tissues obtained from TCGA. As presented in Table III, lower miR-139-5p expression levels were observed in HCC tissues $(n=371)$ than in normal tissues $(\mathrm{n}=49)(6.4189 \pm 1.2376$ vs. $8.5117 \pm 0.4539 ; \mathrm{t}=-22.926$; $\mathrm{P}<0.01)$. Statistically significant associations $(\mathrm{P}<0.05)$ were 
observed among miR-139-5p expression and tumor grade (G1/G2/G3/G4), pathological stage (stage I/II/III/IV) and $\mathrm{T}$ stage (T1-2/3-4). However, no statistically significant association was observed between miR-139-5p expression and the remaining clinicopathological characteristics.

Meta-analysis of miRNA-seq and microarray data from four public databases. As illustrated in Fig. 2, miR-139-5p downregulation in HCC groups was clearly observed in the following datasets: GSE12717, GSE21362, GSE36915, GSE39678, GSE40744, GSE41874, GSE54751, GSE69580, GSE98269, GSE115016 and TCGA. However, higher miR-139-5p expression levels in HCC tissues were identified in the E-MTAB-4809 dataset acquired from ArrayExpress, and no statistically significant difference was detected in the remaining datasets. In addition, corresponding ROC curves were constructed and AUC values were calculated (Fig. 3). Moreover, pooled SMDs with 95\% CIs for all included datasets are presented as a forest plot in Fig. 4A, suggesting reduced miR-139-5p expression in HCC tissues, as determined by a random-effects model $\left(\mathrm{SMD}=-0.84 ; 95 \%\right.$ CI: -1.36 to $-0.32 ; \mathrm{P}<0.001 ; \mathrm{I}^{2}=85.7 \%$ ). Additionally, the Begg's funnel plot of combined datasets revealed clear symmetry, indicating no evident publication bias in the current analysis of miRNA-seq and microarray data (Fig. 4B). Furthermore, the results from the sensitivity analysis (Fig. 4C) demonstrated no indication of any individual study significantly affecting the final SMD and its $95 \% \mathrm{CI}$.

Determination of the diagnostic efficacy of miR-139-5p expression in HCC. As shown in Fig. 5, the combined diagnostic OR was 14.39 (CI: 5.96-34.75) and a slight heterogeneity was indicated by the $\mathrm{I}^{2}$, which was found to be $56.3 \%$. Furthermore, the sensitivity and specificity of the included datasets were calculated, and the corresponding results were 0.87 (0.85-0.90) and 0.66 (0.60-0.72), respectively. Moreover, the pooled PLR and NLR values were 2.50 (CI: 1.53-4.07) and 0.23 (CI: 0.16-0.33), respectively. The AUC result of the sROC curve was 0.8978 , indicating that miR-139-5p low-expression has a relatively high diagnostic efficacy to discriminatee HCC from non-tumor liver tissue based on the miRNA-seq and microarray data.

Clinical role of miR-139-5p expression in HCC tissue samples determined from scientific publications. Four publications $(25,28,29,54)$ offered correlative prognostic information to verify the clinical role of miR-139-5p in HCC. HRs for OS were combined with 95\% CIs (HR=1.37; 95\% CI: 1.07-1.76; $\mathrm{P}=0.001)$ and calculated using a fixed-effects model due to a lack of notable heterogeneity $\left(\mathrm{I}^{2}=43.9 \% ; \mathrm{P}=0.148\right)$. The results indicated that decreased miR-139-5p expression was negatively associated with patient prognosis, as shown in Table IV and Fig. 6A. Furthermore, Begg's funnel test was performed to evaluate publication bias in the four included studies (Fig. 6B). No marked bias was observed based on the symmetry of the funnel plot. Moreover, as shown in Fig. 6C, it was concluded that individual studies did not affect the final outcomes. Only two studies $(25,29)$ provided correlations among clinicopathological parameters and miR-139-5p expression in HCC tissues, while other studies reported on DFS (28) and RFS (29) rates (DFS: HR $=1.67,95 \%$ CI: 0.57-4.87; RFS: HR=1.20, 95\% CI: 0.61-2.37). 
Table II. Essential features of datasets involving miR-139-5p expression founded on GEO, TCGA, Oncomine and Array Express databases.

\begin{tabular}{|c|c|c|c|c|c|c|c|c|c|}
\hline \multirow[b]{2}{*}{ Dataset } & \multicolumn{3}{|c|}{$\mathrm{HCC}$} & \multicolumn{3}{|c|}{ Non-tumor } & \multirow[b]{2}{*}{$\mathrm{t}$} & \multirow[b]{2}{*}{ P-value } & \multirow[b]{2}{*}{ AUC } \\
\hline & $\mathrm{N}$ & M & SD & $\mathrm{N}$ & M & SD & & & \\
\hline GSE12717 & 10 & 5.9442 & 1.33223 & 6 & 8.2381 & 0.44433 & -4.036 & 0.001 & 0.917 \\
\hline GSE21279 & 4 & 3.3278 & 2.49676 & 12 & 3.3192 & 3.49780 & 0.005 & 0.996 & 0.531 \\
\hline GSE21362 & 73 & 4.8902 & 1.51216 & 73 & 6.3078 & 1.02803 & -6.624 & $<0.001$ & 0.833 \\
\hline GSE36915 & 68 & 12.0473 & 1.20972 & 21 & 13.5388 & 0.60042 & -7.583 & $<0.001$ & 0.891 \\
\hline GSE39678 & 16 & 7.9983 & 0.57305 & 8 & 8.7452 & 0.64627 & -2.888 & 0.009 & 0.828 \\
\hline GSE40744 & 26 & 8.5754 & 1.41834 & 50 & 9.4392 & 0.47678 & -3.018 & 0.005 & 0.735 \\
\hline GSE41874 & 3 & 0.8372 & 0.06337 & 4 & 1.1558 & 0.06887 & -6.252 & 0.002 & 1.000 \\
\hline GSE50013 & 14 & 1.7929 & 4.95168 & 13 & 0.9769 & 2.13937 & 0.548 & 0.589 & 0.448 \\
\hline GSE54751 & 10 & 0.0110 & 0.00801 & 10 & 0.0435 & 0.02363 & -4.119 & 0.002 & 0.920 \\
\hline GSE64632 & 3 & 0.3118 & 0.38826 & 3 & 0.1204 & 0.06350 & 0.843 & 0.484 & 0.444 \\
\hline GSE67882 & 3 & 12.1139 & 1.96061 & 8 & 9.9169 & 3.02709 & 1.149 & 0.280 & 0.250 \\
\hline GSE69580 & 5 & 1.3212 & 1.21214 & 5 & 10.8835 & 3.12460 & -6.380 & $<0.001$ & 1.000 \\
\hline GSE98269 & 3 & 5.4040 & 0.25288 & 3 & 5.9628 & 0.14467 & -3.322 & 0.029 & 1.000 \\
\hline GSE115016 & 12 & 9.5731 & 12.11435 & 12 & 48.9893 & 26.09749 & -4.746 & $<0.001$ & 0.944 \\
\hline TCGA & 371 & 6.4189 & 1.23762 & 49 & 8.5117 & 0.45390 & -22.926 & $<0.001$ & 0.953 \\
\hline E-MTAB-511 & 8 & 8.1250 & 10.04899 & 10 & 6.7000 & 9.35771 & 0.311 & 0.760 & 0.450 \\
\hline E-MTAB-3347 & 4 & 6.7444 & 0.34443 & 4 & 6.8057 & 0.38966 & -0.236 & 0.821 & 0.500 \\
\hline E-MTAB-4809 & 12 & 9.2651 & 1.27329 & 12 & 5.1714 & 1.42375 & 7.424 & $<0.001$ & 0.000 \\
\hline
\end{tabular}

GEO, Gene Expression Omnibus; TCGA, The Cancer Genome Atlas; N, number; M, mean; SD, standard deviation; AUC, area under the curve; HCC, hepatocellular carcinoma.

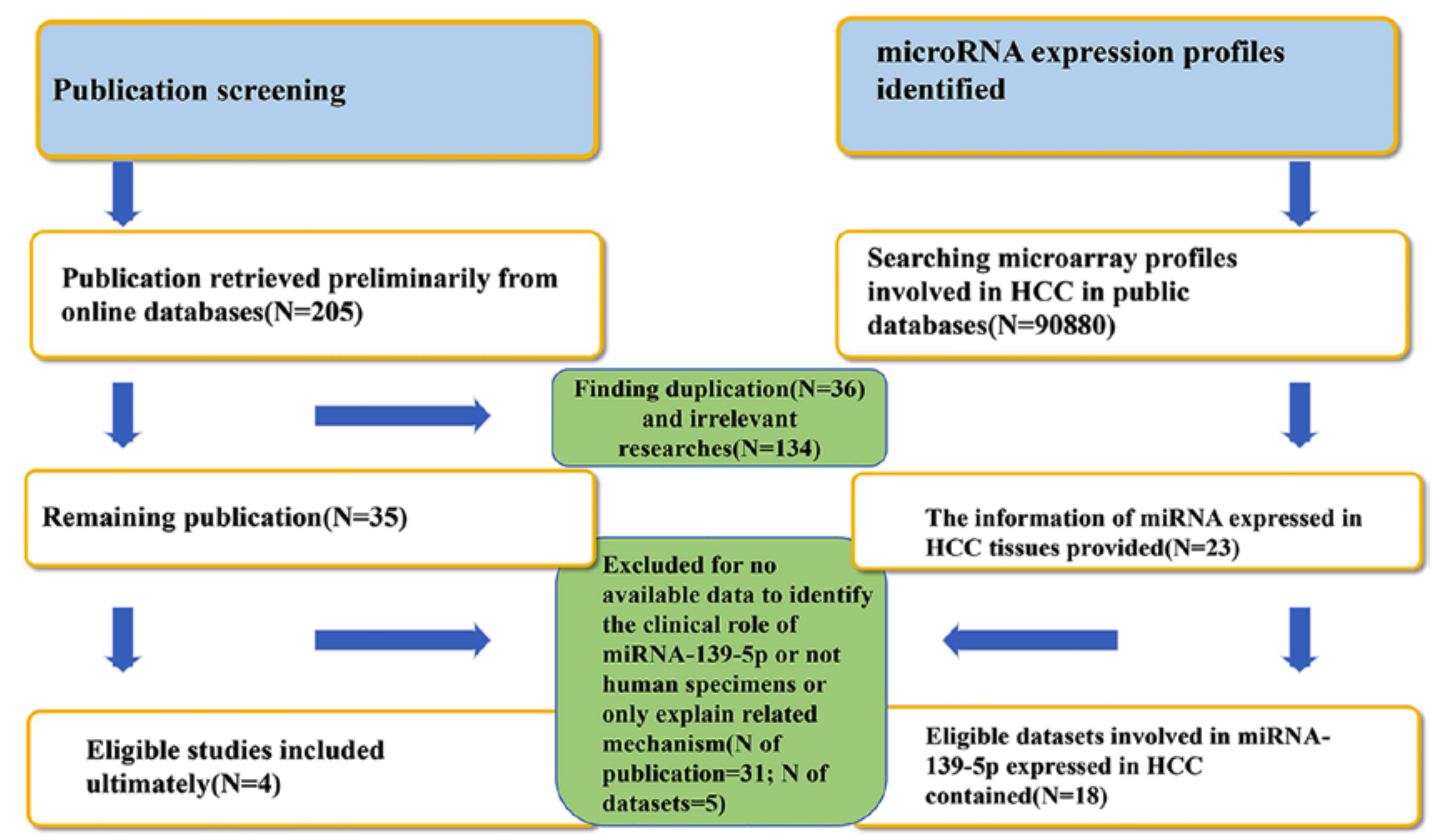

Figure 1. Flow chart illustrating the process for selecting published studies and microarray data. HCC, hepatocellular carcinoma; miRNA, microRNA.

\section{Bioinformatics analysis}

Target genes of miR-139-5p and their clinical roles. A total of 4,294 differentially expressed genes obtained from TCGA, 1,406 targeted genes predicted by the miRWalk website, and a total of 382 intersecting genes were downloaded as final target genes (Fig. 7A). Furthermore, DAVID was employed to conduct a GO functional enrichment analysis, in addition to KEGG and Panther pathway analyses for 
Table III. Clinicopathological parameters of miRNA-139-5p regulation in HCC tissues from TCGA.

\begin{tabular}{|c|c|c|c|c|}
\hline \multirow[b]{2}{*}{ Clinicopathological features } & \multirow[b]{2}{*}{$\mathrm{n}$} & \multicolumn{3}{|c|}{ MiR-139-5p relevant expression $\left(\log _{2} x\right)$} \\
\hline & & Mean \pm SD & $\mathrm{t}$ & P-value \\
\hline Tissue & & & -22.926 & $<0.01$ \\
\hline $\mathrm{HCC}$ & 371 & $6.4189 \pm 1.2376$ & & \\
\hline Normal tissue & 49 & $8.5117 \pm 0.4539$ & & \\
\hline Age (years) & & & -0.059 & 0.953 \\
\hline$\geq 60$ & 200 & $6.5022 \pm 1.4621$ & & \\
\hline$<60$ & 170 & $6.5107 \pm 1.3107$ & & \\
\hline Sex & & & 1.196 & 0.233 \\
\hline Female & 119 & $6.4136 \pm 1.4532$ & & \\
\hline Male & 251 & $6.5982 \pm 1.2365$ & & \\
\hline Race & & & $\mathrm{F}=0.662$ & 0.576 \\
\hline White & 181 & $6.5850 \pm 1.4719$ & & \\
\hline Black or African American & 17 & $6.6571 \pm 0.9780$ & & \\
\hline Asian & 161 & $6.4148 \pm 1.2035$ & & \\
\hline American Indian or & & & & \\
\hline Alaska native & 2 & $5.9092 \pm 2.1116$ & & \\
\hline Cancer status & & & -1.605 & 0.109 \\
\hline Tumor free & 202 & $6.6343 \pm 1.3739$ & & \\
\hline With tumor & 150 & $6.3997 \pm 1.3318$ & & \\
\hline Grade & & & $\mathrm{F}=8.305$ & $<0.001$ \\
\hline G1 & 55 & $7.1548 \pm 1.1886$ & & \\
\hline G2 & 173 & $6.6150 \pm 1.3285$ & & \\
\hline G3 & 124 & $6.1845 \pm 1.2038$ & & \\
\hline G4 & 13 & $6.1198 \pm 1.1988$ & & \\
\hline Pathological stage & & & $\mathrm{F}=7.575$ & $<0.001$ \\
\hline Stage I & 172 & $6.8524 \pm 1.1347$ & & \\
\hline Stage II & 85 & $6.2293 \pm 1.3337$ & & \\
\hline Stage III & 85 & $6.1721 \pm 1.4300$ & & \\
\hline Stage IV & 5 & $6.3922 \pm 1.6006$ & & \\
\hline T stage & & & -2.595 & 0.010 \\
\hline $\mathrm{T} 1-2$ & 275 & $6.6139 \pm 1.3039$ & & \\
\hline T3-4 & 93 & $6.1970 \pm 1.4403$ & & \\
\hline Node involvement & & & 1.506 & 0.133 \\
\hline No & 232 & $6.3513 \pm 1.5065$ & & \\
\hline Yes & 4 & $7.4924 \pm 1.1574$ & & \\
\hline Metastasis & & & -0.554 & 0.580 \\
\hline No & 267 & $6.4695 \pm 1.3007$ & & \\
\hline Yes & 4 & $6.1048 \pm 1.6927$ & & \\
\hline Survival status & & & -1.756 & 0.080 \\
\hline Dead & 129 & $6.3757 \pm 1.4070$ & & \\
\hline Alive & 241 & $6.6261 \pm 1.2509$ & & \\
\hline
\end{tabular}

HCC, hepatocellular carcinoma; TCGA, The Cancer Genome Atlas; N, number; SD, standard deviation; F, One-way analysis of variance (ANOVA) test was performed to assess the difference for $>2$ groups.

the purpose of identifying specific molecular mechanisms of miR-139-5p related to HCC progression and prognosis. As shown in Fig. 8 and Table V, the genes were significantly enriched in 'response to drug', 'signal transduction', 'cell migration involved in sprouting angiogenesis' and 'positive regulation of cell proliferation' $(\mathrm{P}<0.01)$, based on the biological process of GO. Regarding the cellular component of GO, the genes were specifically focused on 'plasma 

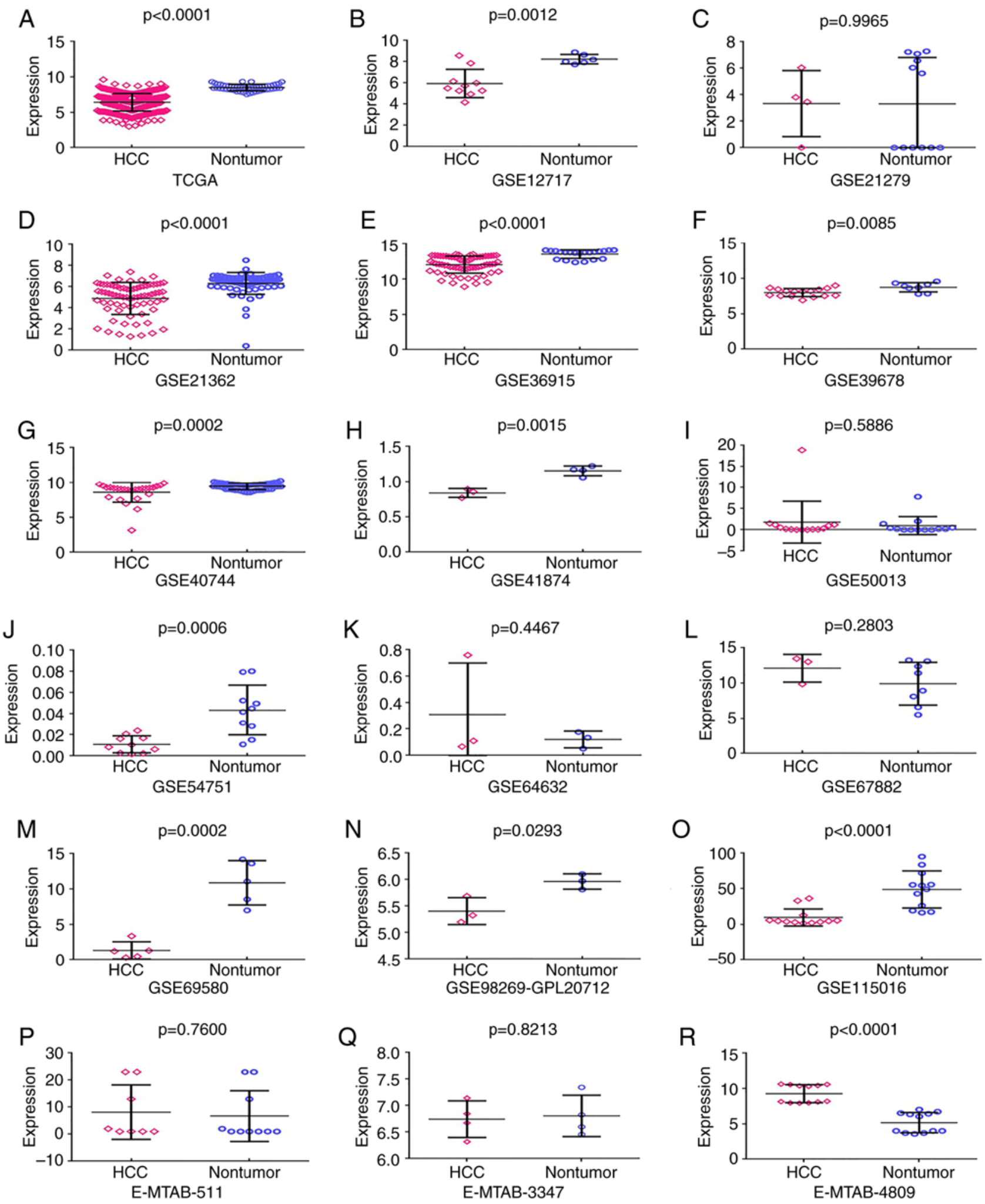

Figure 2. Differential expressive situation of miR-139-5p between HCC and nontumor liver tissues established using 18 microarray and miRNA sequencing datasets. (A) TCGA; (B) GSE12717; (C) GSE21279; (D) GSE21362; (E) GSE36915; (F) GSE39678; (G) GSE40744; (H) GSE41874; (I) GSE50013; (J) GSE54751; (K) GSE64632; (L) GSE67882; (M) GSE69580; (N) GSE98269-GPL20712; (O) GSE115016; (P) E-MTAB-511; (Q) E-MTAB-3347; (R) E-MTAB-4809. HCC, hepatocellular carcinoma; TCGA, The Cancer Genome Atlas; miR, microRNA.

membrane', 'integral component of plasma membrane' and 'neuron projection' $(\mathrm{P}<0.01)$. Concerning the molecular function of GO, the functional genes were primarily assembled in 'transcription factor activity, sequence-specific DNA binding' and 'Ras guanyl-nucleotide exchange factor activity' $(\mathrm{P}<0.01)$. Moreover, as presented in Table VI, KEGG and Panther pathway analyses were performed on several signaling pathways, including 'pathways in cancer', 'Ras signaling pathway' and 'endogenous cannabinoid signaling' $(\mathrm{P}<0.05)$. 
A
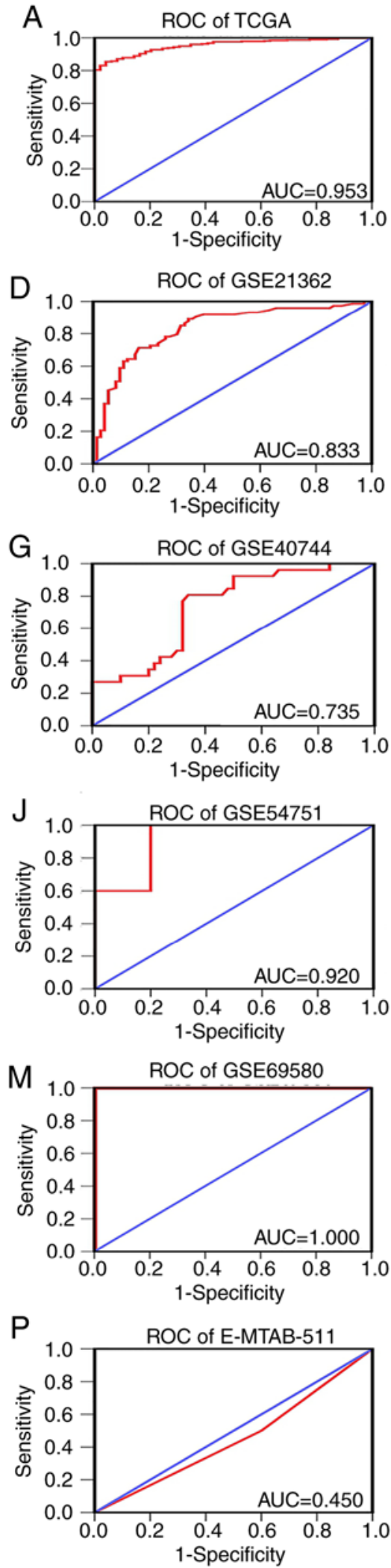
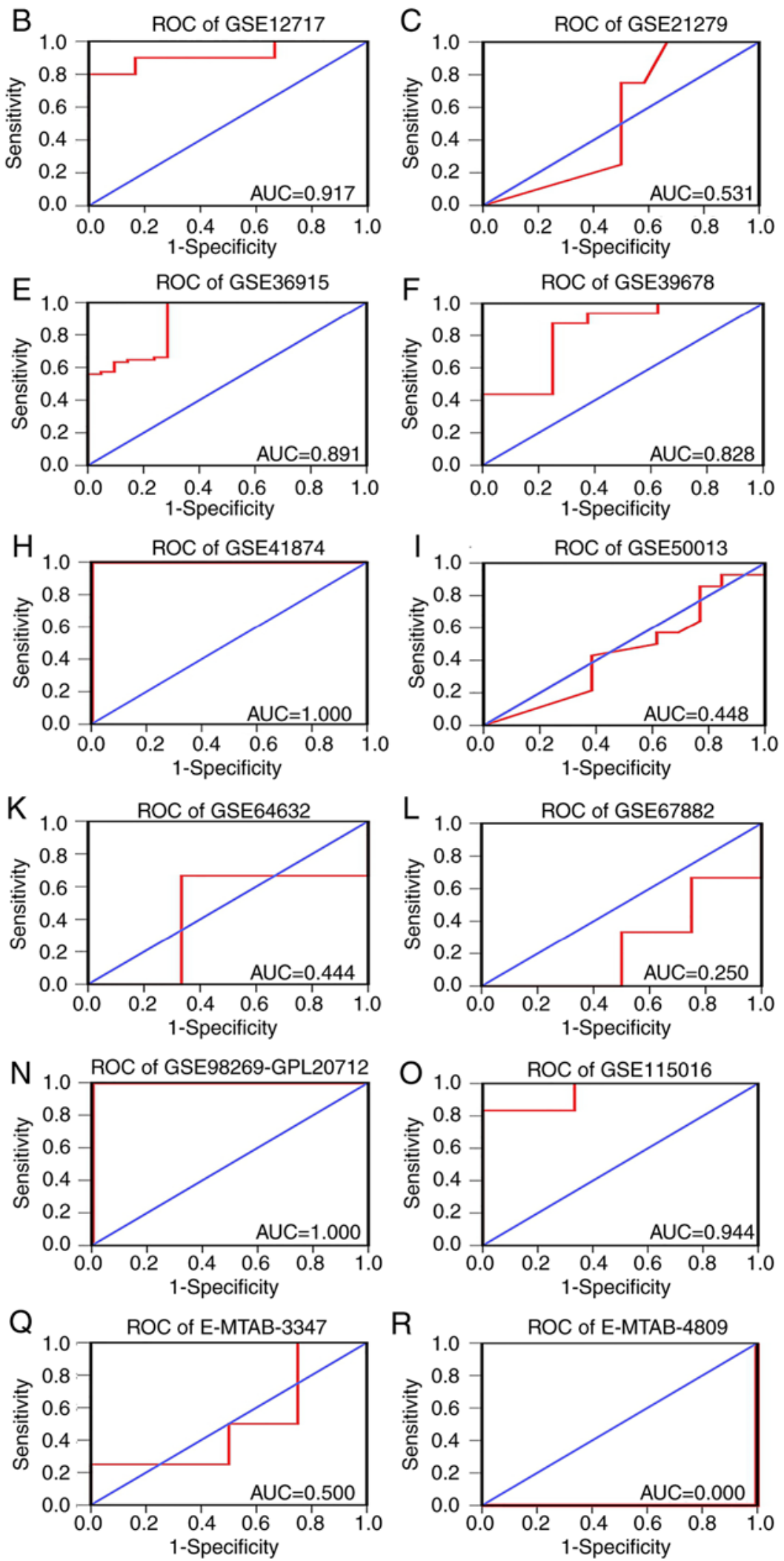

Figure 3. ROC curve of miR-139-5p regulation in HCC and nontumor liver tissues in each analyzed dataset. (A) TCGA; (B) GSE12717; (C) GSE21279; (D) GSE21362; (E) GSE36915; (F) GSE39678; (G) GSE40744; (H) GSE41874; (I) GSE50013; (J) GSE54751; (K) GSE64632; (L) GSE67882; (M) GSE69580; (N) GSE98269-GPL20712; (O) GSE115016; (P) E-MTAB-511; (Q) E-MTAB-3347; (R) E-MTAB-4809. HCC, hepatocellular carcinoma; ROC, receiver operating characteristic; TCGA, The Cancer Genome Atlas; miR, microRNA.

Construction of target gene networks and identification of hub genes. The STRING database was employed in order to predict 382 intersected genes that were associated with miR-139-5p expression by constructing a PPI network. As presented in Fig. 7B, the top eight genes, namely, FBJ murine osteosarcoma viral oncogene homolog (FOS), chromatin licensing and 


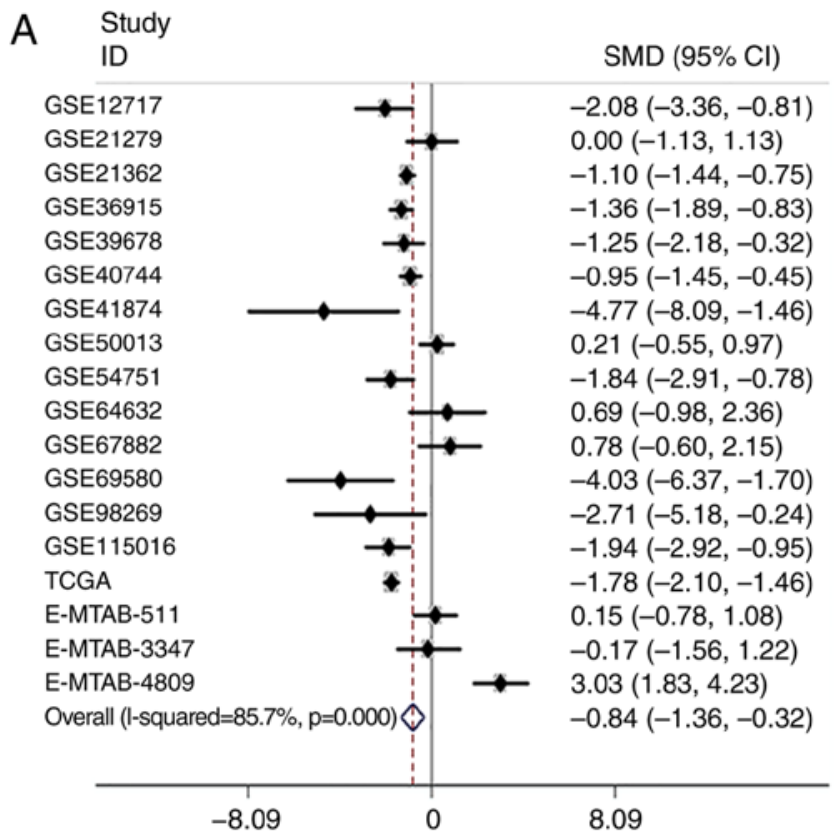

B Begg's funnel plot with pseudo $95 \%$ confidence limits

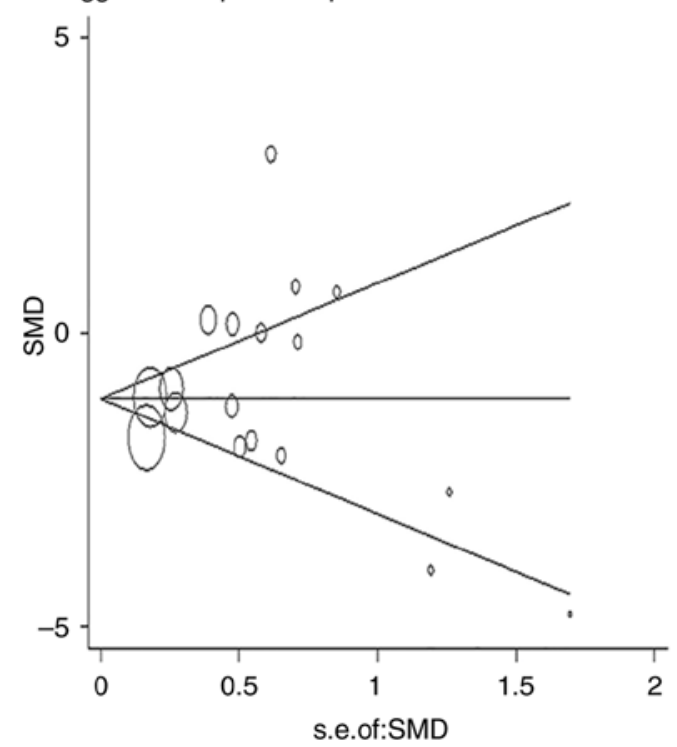

C Meta-analysis estimates, given named study is omittied

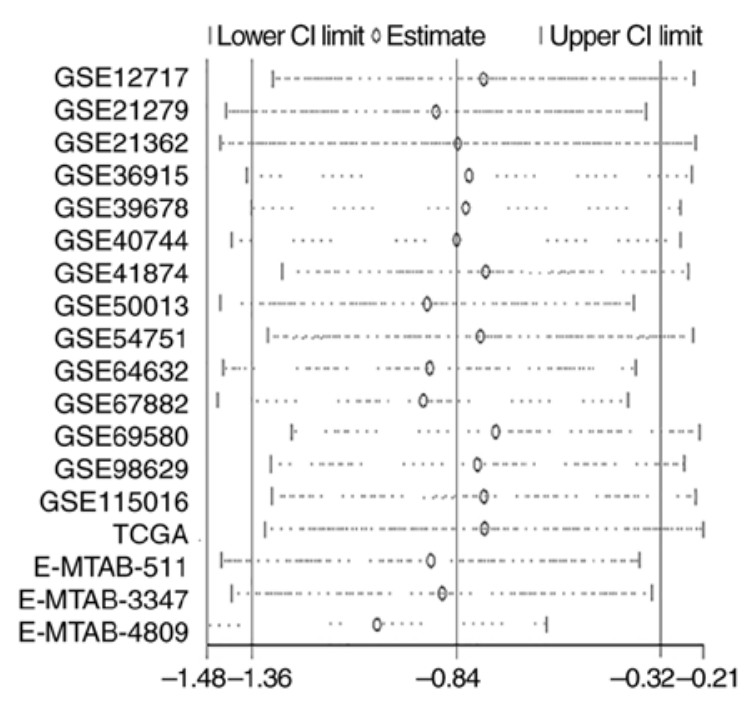

Figure 4. Integrated meta-analysis designed to identify the miR-139-5p expression levels in 18 analyzed datasets including HCC and nontumor liver tissues. (A) Forest plot showing that miR-139-5p is downregulated in HCC (SMD=-0.84; 95\% CI: -1.36 to $-0.32 ; \mathrm{P}<0.001 ; \mathrm{I}^{2}=85.7 \%$ ). (B) Symmetry of the funnel plot revealed no evident publication bias among the 18 included datasets. The size of each circle represents the sample size of each dataset. (C) Sensitivity analysis showing the stability of the 18 included datasets. HCC, hepatocellular carcinoma; SMD, standard mean difference; CI, confidence interval; miR, microRNA; $\mathrm{I}^{2}$, inconsistency index.

DNA replication factor 1 (CDT1), enhancer of zeste homolog 2 (EZH2), recombination protein (RAD51), forkhead box M1 (FOXM1), forkhead box O1 (FOXO1), cell division cycle 25A (CDC25A), and guanine nucleotide binding protein alpha 12 (GNA12) with connection degrees $>14$ were chosen from the PPI network for further investigation. Moreover, four genes, FOS, FOXO1, GNA12 and RAD51, identified in the first signaling pathway termed 'pathways in cancer' were eventually determined as hub genes.

Target gene expression and associated clinical implications. An analysis was conducted using GEPIA to determine the top eight genes associated with HCC development and verify their specific clinical implications. The expression levels of four genes, CDT1, EZH2, FOXM1 and RAD51, were substantially increased in HCC tissues, whereas decreased FOS gene expression was observed in HCC tissues $(\mathrm{P}<0.05$; Fig. 9). Moreover, the prognostic value of the top eight genes was also evaluated (Fig. 10). The expression of six genes, CDT1, EZH2, RAD51, FOXM1, GNA12 and CDC25A, was negatively associated with the OS of patients with HCC. In addition, the frequency of mutations in the top eight genes (Fig. 11), and the expression patterns of the four hub genes FOS, FOXO1, GNA12 and RAD51 in HCC and normal liver tissues were explored using the Human Protein Atlas database (Fig. 12). Furthermore, based on TCGA data, a positive correlation was identified 
A

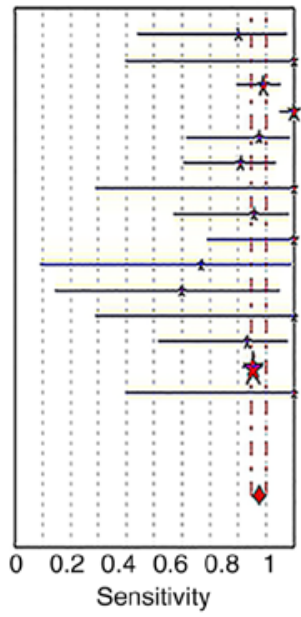

C

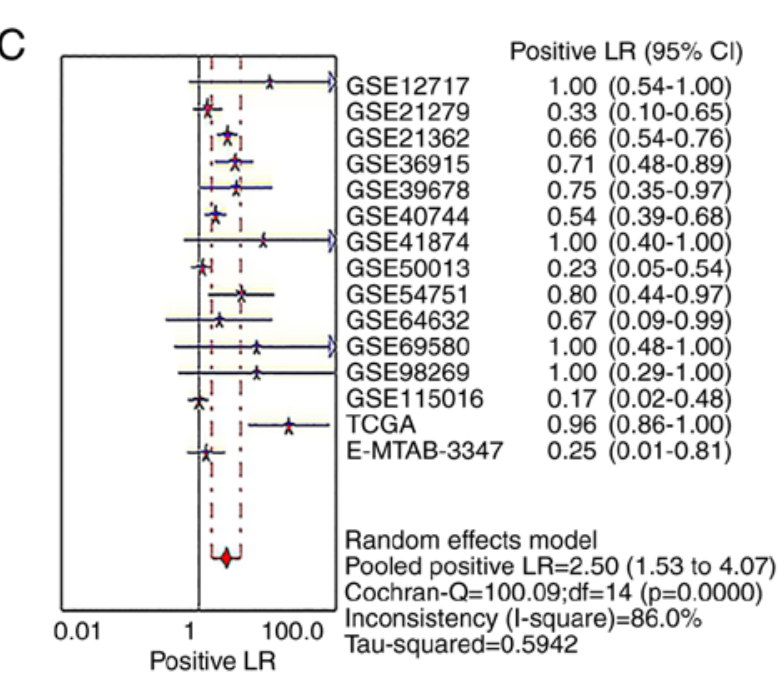

Sensitivity $(95 \% \mathrm{Cl})$

$1.00(0.54-1.00)$

$0.33(0.10-0.65$

$0.66(0.54-0.76)$

$0.71(0.48-0.89)$

$0.75(0.35-0.97)$

$0.54(0.39-0.68)$

$1.00(0.40-1.00$

$0.23(0.05-0.54$

$0.80(0.44-0.97)$

$0.67(0.09-0.99)$

$1.00(0.48-1.00)$

$1.00(0.29-1.00)$

$\begin{array}{ll}\text { GSE115016 } & 0.17(0.02-0.48) \\ \text { TCGA } & 0.96(0.86-1.00) \\ \text { E-MTAB-3347 } & 0.25(0.01-0.81)\end{array}$

$\begin{array}{ll}\text { GSE115016 } & 0.17(0.02-0.48) \\ \text { TCGA } & 0.96(0.86-1.00) \\ \text { E-MTAB-3347 } & 0.25(0.01-0.81)\end{array}$

Pooled sensitivity $=0.87$ ( 0.85 to 0.90$)$

Chi-square $=30.97 ; \mathrm{df}=14 \quad(\mathrm{p}=0.0056)$ Inconsistency (1-square) $=54.8 \%$

Sensitivity
B

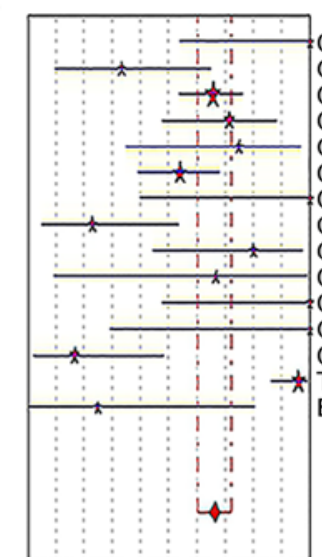

GSE12717 GSE21279 GSE21362 GSE 36915

GSE39678 GSE40744 GSE41874 GSE50013 GSE54751 GSE64632 GSE69580 GSE98269 TCGA

Specificity $(95 \% \mathrm{Cl})$

$1.00(0.54-1.00)$

$0.33(0.10-0.65$

$0.66(0.54-0.76$

$0.71(0.48-0.89$

$0.75(0.35-0.97)$

$0.54(0.39-0.68)$

$1.00(0.40-1.00)$

$0.23(0.05-0.54)$

$0.80(0.44-0.97)$

$0.67(0.09-0.99$

$1.00(0.48-1.00)$

$1.00(0.29-1.00)$

$0.17(0.02-0.48$

$0.17(0.02-0.48$

$0.96(0.86-1.00)$

$0.25(0.01-0.81)$

Pooled specificity $=0.66$ ( 0.60 to 0.72$)$

Chi-square $=76.79 ; \mathrm{df}=14 \quad(\mathrm{p}=0.0000)$

$\begin{array}{lllllll}0 & 0.2 & 0.4 & 0.6 & 0.8 & 1\end{array}$

Sensitivity

D

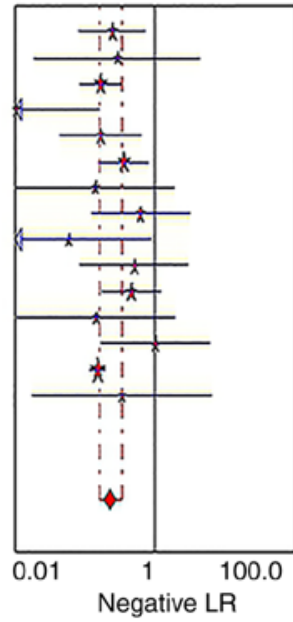

GSE 12717

GSE21279

GSE21362

GSE36915

GSE39678

GSE40744

GSE41874

GSE50013

GSE54751

GSE64632

GSE69580

GSE98269

GSE115016

TCGA

E-MTAB-3347

Negative LR $(95 \% \mathrm{Cl})$

$1.00(0.54-1.00)$

$0.33(0.10-0.65)$

$0.66(0.54-0.76$

$0.71(0.48-0.89)$

$0.75(0.35-0.97)$

$0.54(0.39-0.68)$

$1.00(0.40-1.00)$

$0.23(0.05-0.54)$

$0.80(0.44-0.97)$

$0.67(0.09-0.99)$

$1.00(0.48-1.00)$

$1.00(0.29-1.00)$

$0.17(0.02-0.48)$

$0.96(0.86-1.00)$

$0.25(0.01-0.81)$

Random effects mode

Pooled negative LR $=0.23$ (0.16 to 0.33 )

Cochran- $\mathrm{Q}=20.28 ; \mathrm{df}=14(\mathrm{p}=0.1217)$

Inconsistency (I-square) $=31.0 \%$

Tau-squared $=0.1287$
E

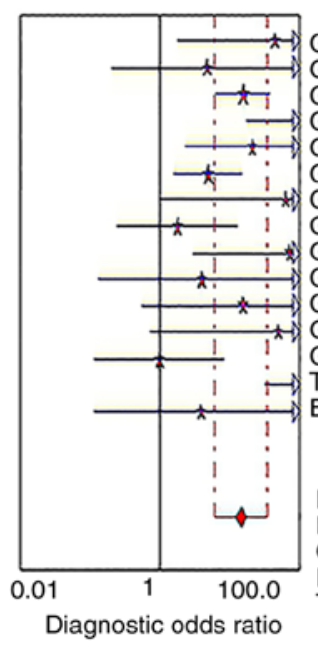

Diagnostic OR (95\% Cl)

$1.00(0.54-1.00)$

$0.33(0.10-0.65$

$0.66(0.54-0.76)$

$0.71(0.48-0.89)$

$0.75(0.35-0.97)$

$0.54(0.39-0.68)$

$1.00(0.40-1.00)$

$0.23(0.05-0.54$

$0.80(0.44-0.97)$

$0.67(0.09-0.99)$

$1.00(0.48-1.00)$

$1.00(0.29-1.00)$

$0.17(0.02-0.48)$

$0.96(0.86-1.00)$

$0.25(0.01-0.81)$

E-MTAB-3347

Random effects mode

Pooled diagnostic odds ratio=14.39 (5.96 to 34.75$)$

Cochran $-\mathrm{Q}=32.01 ; \mathrm{df}=14(\mathrm{p}=0.0040)$

Inconsistency $(\mathrm{I}$-square $)=56.3 \%$

Tau-squared $=1.3971$

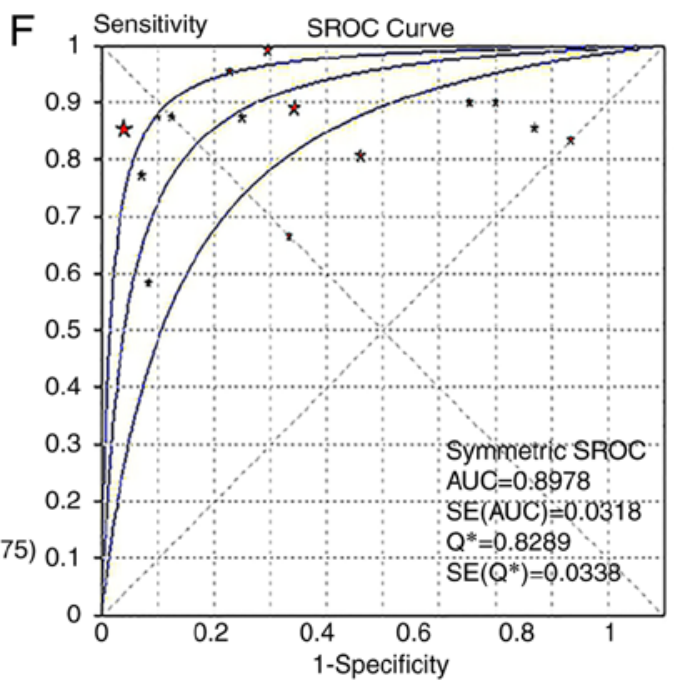

Figure 5. Significance of miR-139-5p expression in the diagnosis of HCC compared with non-tumor liver tissues in 18 datasets. (A) Merged sensitivity was 0.87 (0.85-0.90). (B) Merged specificity was $0.66(0.60-0.72)$. (C) Pooled positive LR was 2.50 (CI: 1.53-4.07). (D) Pooled negative LR was 0.23 (CI: 0.16-0.33). (E) Pooled diagnostic OR was 14.39 (CI: 5.96-34.75). (F) AUC of the sROC curve was 0.8978. HCC, hepatocellular carcinoma; LR, likelihood ratio; $\mathrm{CI}$, confidence interval; OR, odds ratio; AUC, area under the curve; sROC, summary receiver operating characteristic; miR, microRNA.

between miR-139-5p expression in HCC and the regulation of FOS and FOXO1 genes, which were also reduced in HCC tissues (Fig. 13). Consistent with the aforementioned findings, a negative correlation was detected between miR-139-5p expression and the regulation of GNA12 and RAD51 in patients with HCC $(\mathrm{P}<0.001)$. 


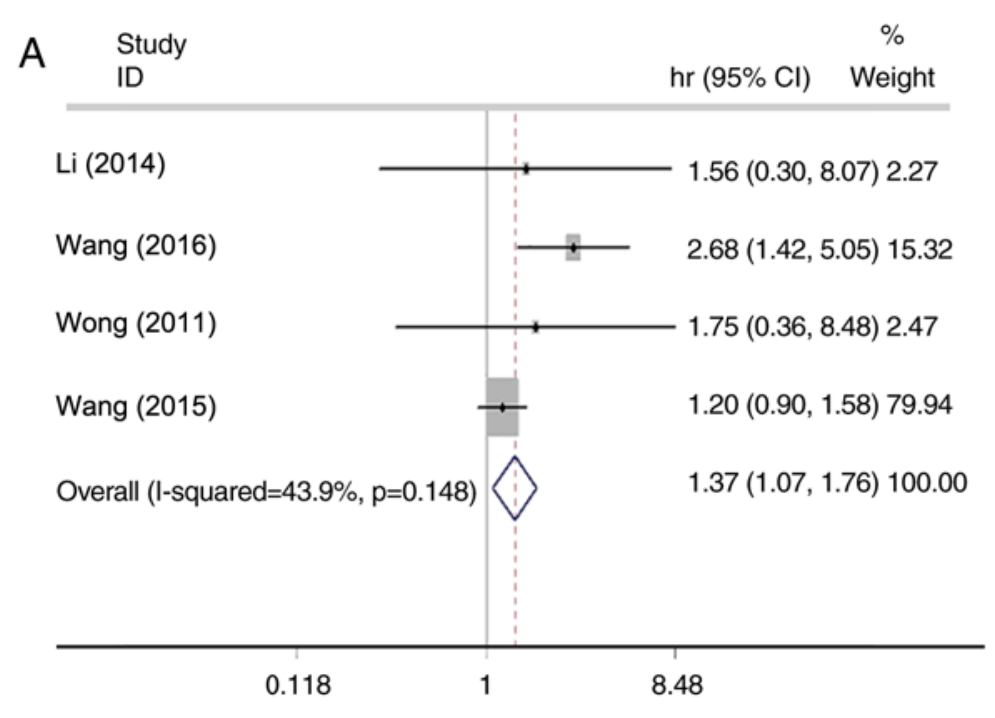

B

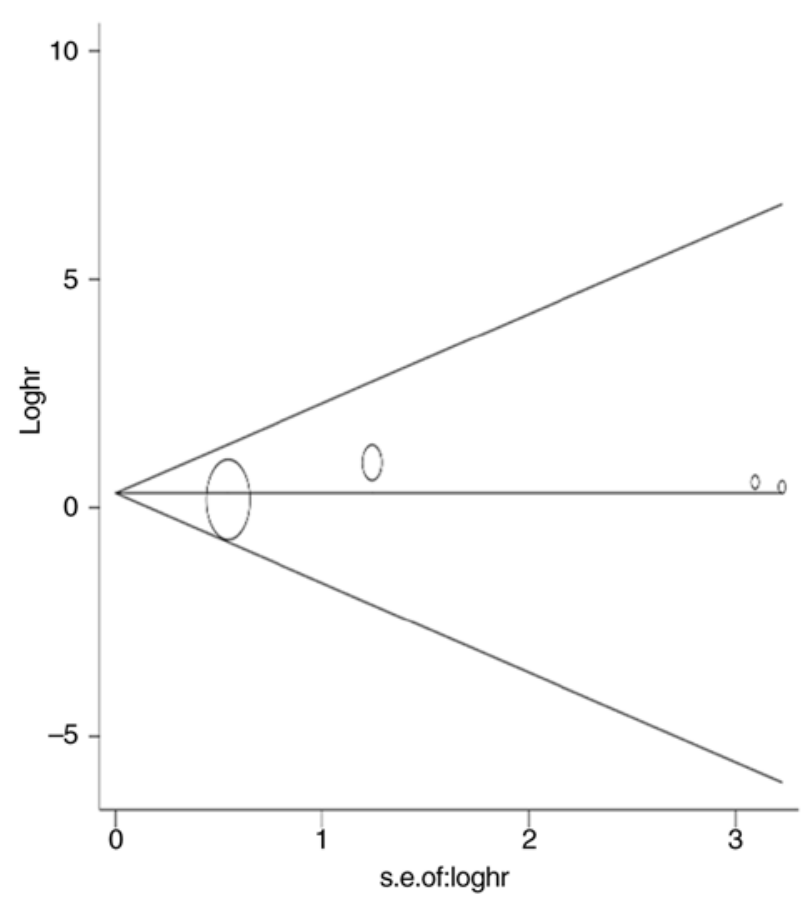

C Meta-analysis estimates, given named study is omitted

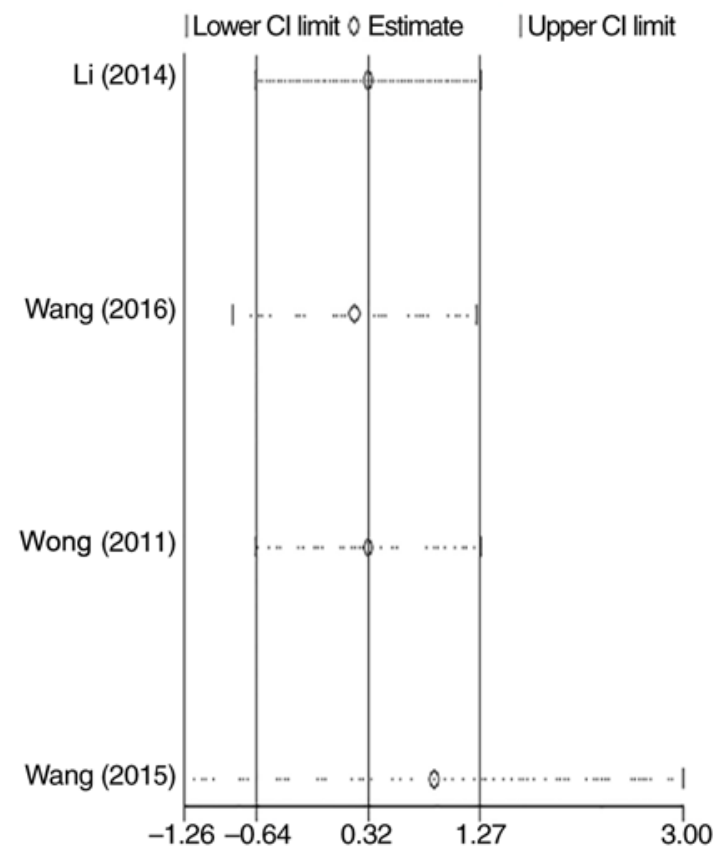

Figure 6. Integrated meta-analysis for determining miR-139-5p expression in HCC. (A) Forest plot demonstrating a poor prognosis for patients with HCC presenting reduced miR-139-5p expression based on four studies reporting the OS of patients with HCC. (B) Symmetry of the funnel plot revealed no evident publication bias among the four publications. The size of the circle represents the sample size of each study. (C) Sensitivity analysis shows the stability of the four publications. HCC, hepatocellular carcinoma; OS, overall survival; CI, confidence interval; miR, microRNA.

\section{Discussion}

Based on the miRNA-seq and microarray data retrieved from four public databases and data obtained from published literature $(25,28,29,54)$, including human HCC specimens, the primary aim of this study was to perform additional bioinformatics analyses examining miR-139-5p and the corresponding target gene expression and molecular mechanism involved in HCC development. Based on the analytical miRNA-seq and microarray data, reduced miR-139-5p expression levels were observed in the HCC group (SMD=-0.84; 95\% CI: -1.36 to $-0.32 ; \mathrm{P}<0.001)$. Based on the data retrieved from the TCGA database, certain clinicopathological features, including grade, pathological and T stage, were associated with miR-139-5p expression, indicating that decreased miR-139-5p expression may facilitate disease progression. Moreover, the AUC value of the sROC curve for data obtained from public databases was 0.8978 , indicating a relatively high diagnostic efficacy of miR-139-5p expression. Regarding the association between clinical characteristics and miR-139-5p expressed in HCC based on relevant literature, the pooled OS (HR=1.37; 95\% CI: 1.07-1.76) revealed that decreased miR-139-5p expression led to a poor prognosis in patients with HCC. Furthermore, bioinformatics software was used to identify 382 genes regulated by miR-139-5p in HCC, for which GO, KEGG and Panther pathway analyses were performed. The target genes 
Table IV. Merged HRs and corresponding 95\% CIs for OS in patients with HCC obtained using a meta-analysis based on fixed-effects model.

\begin{tabular}{lcccccccc}
\hline Study & Year & HR & LL & UL & $\mathrm{p}$ & $\mathrm{I}^{2}(\%)$ & P-value & (Refs.) \\
\hline Li et al & 2014 & 1.56 & 0.30 & 8.07 & & & $(54)$ \\
Wang et al & 2016 & 2.68 & 1.42 & 5.05 & & & & $(25)$ \\
Wong et al & 2011 & 1.75 & 0.36 & 8.48 & & & & $(29)$ \\
Wang et al & 2015 & 1.20 & 0.91 & 1.58 & & & & $(28)$ \\
Pooled & & 1.37 & 1.07 & 1.76 & 0.001 & 43.9 & 0.148 & \\
\hline
\end{tabular}

$\mathrm{P}, \mathrm{P}<0.05$ and the $95 \% \mathrm{CI}$ not exceeding 1 indicated HR was considered significant $(\mathrm{HR}=1.37 ; 95 \% \mathrm{CI}: 1.07-1.76 ; \mathrm{P}=0.001) ; \mathrm{P}, \mathrm{P}>0.05$ indicated no significant heterogeneity was observed using fixed-effects mode $\left(\mathrm{P}=0.1481, \mathrm{I}^{2}=43.9 \%\right)$. HR, hazard ratio; $\mathrm{CI}$, confidence interval; OS, overall survival; HCC, hepatocellular carcinoma; LL, lower confidence limit; UL, upper confidence limit; $\mathrm{I}^{2}$, inconsistency index;
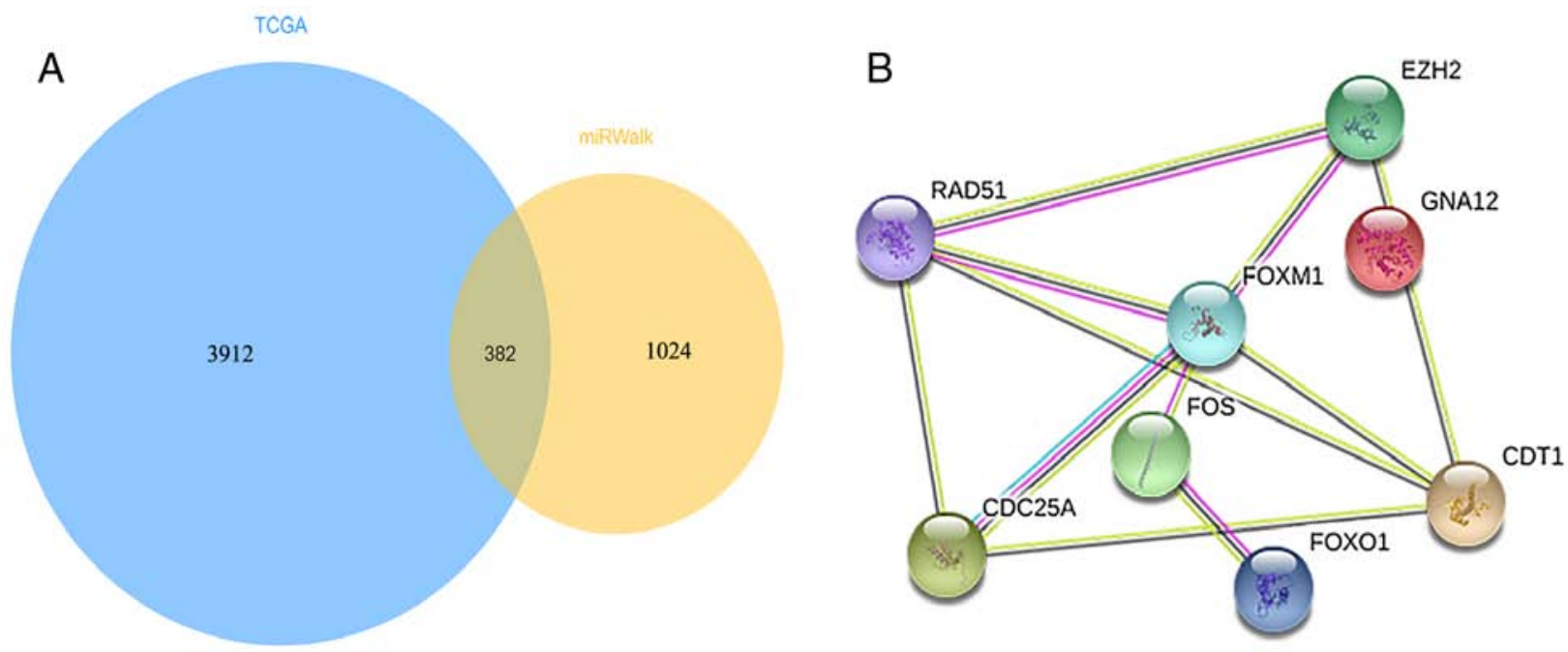

Figure 7. Process for determining predicted downstream target genes. (A) Venn diagram presenting the 4,294 differentially expressed genes obtained from TCGA and 1,406 targeted genes predicted by the miRWalk website; 382 intersecting genes were ultimately identified. (B) Performance of the protein-protein network for validating the connections among the top eight target genes. TCGA, The Cancer Genome Atlas.

were significantly enriched in 'response to drug', 'signal transduction', 'plasma membrane', 'transcription factor activity, sequence-specific DNA binding', 'pathways in cancer', 'Ras signaling pathway' and 'endogenous cannabinoid signaling' $(\mathrm{P}<0.05)$. In addition, the top eight target genes, FOS, CDT1, EZH2, RAD51, FOXM1, FOXO1, GNA12 and CDC25A, were selected by PPI network construction; and their clinical impacts were investigated. Moreover, FOS, FOXO1, GNA12 and RAD51, which were enriched in 'pathways in cancer', were determined as hub genes. The IHC staining for proteins encoded by these four genes, namely, FOS, FOXO1, GNA12 and RAD51, was further confirmed using the Human Protein Atlas database, and the expression atlas of the pathological sections supported the downregulation of FOS and FOXO1 accompanied by the upregulation of GNA12 and RAD51 in HCC tissues. Furthermore, a relativity analysis revealed that miR-139-5p expression was positively correlated with the regulation of FOS and FOXO1 and negatively correlated with GNA12 and RAD51 regulation, suggesting that miR-139-5p may mediate the expression of hub genes involved in the aforementioned signaling pathways, mainly in 'pathways in cancer'.
Mature miRNAs, which associate with the RNA-induced silencing complex (RISC), are capable of promoting the interaction of RISC with the 3'-UTR of the downstream target mRNA, resulting in abnormal translation of downstream mRNA. In addition, miR-139-5p is regarded as a tumor suppressor due to its regulatory effects on multiple malignant tumors. Notably, miR-139-5p controls tumor progression by regulating target mRNA expression and participates in downstream signaling pathways in malignancies (55-59), including HCC. According to a report by Wong et al (29), the progression and invasion of HCC are inhibited by miR-139-5p via its effects on Rho-kinase 2 expression. In addition, Au et al (60) revealed that miR-139-5p greatly affects the metastasis-related pathways of HCC cells by increasing the expression of EZH2, which is involved in sustaining the transcriptional suppression of genes over sequential cell generations. The increased expression of EZH2 contributed to metastasis, and poor prognosis of $\mathrm{HCC}$ identified in our analysis (Figs. 9 and 10). As reported by Hua et al (24), miR-139-5p regulates aerobic glycolysis, cell proliferation and motility by interacting with ETS1 in HCC cells. Moreover, a meta-analysis based on published articles that explored 
Table V. Enrichment of downstream target genes regulated by miR-139-5p in Gene Ontology terms in the categories of biological processes, cellular components and molecular functions ${ }^{\mathrm{a}}$.

\begin{tabular}{|c|c|c|}
\hline Term & Counts & P-value \\
\hline \multicolumn{3}{|l|}{ Biological process } \\
\hline Response to drug & 16 & $1.19 \times 10^{-03}$ \\
\hline Signal transduction & 39 & $1.72 \times 10^{-03}$ \\
\hline Cell migration involved in sprouting angiogenesis & 4 & $2.97 \times 10^{-03}$ \\
\hline Positive regulation of cell proliferation & 19 & $5.81 \times 10^{-03}$ \\
\hline Positive regulation of neuron projection development & 7 & $8.64 \times 10^{-03}$ \\
\hline Positive regulation of GTPase activity & 21 & $9.52 \times 10^{-03}$ \\
\hline Regulation of insulin secretion & 6 & $1.07 \times 10^{-02}$ \\
\hline Response to insulin & 6 & $1.07 \times 10^{-02}$ \\
\hline Activation of protein kinase activity & 5 & $1.21 \times 10^{-02}$ \\
\hline Positive regulation of transcription, DNA-templated & 19 & $1.52 \times 10^{-02}$ \\
\hline \multicolumn{3}{|l|}{ Cellular component } \\
\hline Plasma membrane & 111 & $1.30 \times 10^{-04}$ \\
\hline Integral component of plasma membrane & 48 & $2.12 \times 10^{-04}$ \\
\hline Neuron projection & 15 & $2.35 \times 10^{-04}$ \\
\hline Integral component of membrane & 125 & $3.30 \times 10^{-03}$ \\
\hline Neuronal cell body & 14 & $9.02 \times 10^{-03}$ \\
\hline External side of plasma membrane & 11 & $9.07 \times 10^{-03}$ \\
\hline Postsynaptic membrane & 10 & $2.27 \times 10^{-02}$ \\
\hline Anchored component of membrane & 7 & $2.30 \times 10^{-02}$ \\
\hline $\mathrm{Z}$ disc & 7 & $2.77 \times 10^{-02}$ \\
\hline Terminal bouton & 5 & $3.24 \times 10^{-02}$ \\
\hline \multicolumn{3}{|l|}{ Molecular function } \\
\hline Transcription factor activity, sequence-specific DNA binding & 36 & $4.37 \times 10^{-04}$ \\
\hline Ras guanyl-nucleotide exchange factor activity & 8 & $8.28 \times 10^{-03}$ \\
\hline Steroid binding & 4 & $1.62 \times 10^{-02}$ \\
\hline mRNA 3'-UTR binding & 5 & $1.63 \times 10^{-02}$ \\
\hline Sequence-specific DNA binding & 19 & $1.65 \times 10^{-02}$ \\
\hline $\begin{array}{l}\text { Transcriptional activator activity, RNA polymerase II core promoter proximal region } \\
\text { sequence-specific binding }\end{array}$ & 11 & $2.05 \times 10^{-02}$ \\
\hline Kinase activity & 11 & $2.33 \times 10^{-02}$ \\
\hline Pyridoxal phosphate binding & 5 & $2.69 \times 10^{-02}$ \\
\hline DNA binding & 45 & $3.55 \times 10^{-02}$ \\
\hline Calmodulin binding & 9 & $3.62 \times 10^{-02}$ \\
\hline
\end{tabular}

${ }^{\mathrm{a}} \mathrm{P}<0.05$. GO, Gene Ontology.

miR-139-5p expression in digestive system tumors, including one HCC case that was analyzed prospectively, indicated an unfavorable effect on the life expectancy of patients with gastrointestinal tumors (27). Additionally, downregulation of miR-139-5p has been demonstrated to result in poor outcome and disease progression $(25,26,28)$. This finding was confirmed in the present study as indicated by the merged results with an SMD of -0.84 and HR for OS of 1.37 (95\% CI: 1.07-1.76).

To further investigate the oncogenic molecular mechanism of miR-139-5p expression, bioinformatics analysis was performed with biological software and the top eight target genes were ultimately evaluated. Regarding the GO enrichment analysis, target genes were notably enriched in 'response to drug' and 'positive regulation of cell proliferation'. Certain studies have reported that miR-139-5p expression has a major impact on pesticide effects in cancer therapy $(58,61)$. Yoon et al (62) selected certain serum miRNAs, including miR-139-5p, associated with positive radiological responses or improved survival to assess the efficacy of sorafenib in patients with HCC. However, no statistically significant difference was reported between miR-139-5p expression and sorafenib treatment in patients with HCC. Additional correlative studies focused on drug efficacy and miR-139-5p expression should be further developed.

In terms of the pathway analysis, 'pathways in cancer', 'Ras signaling pathway' and 'endogenous cannabinoid 
Table VI. Downstream predicted genes of miR-139-5p are principally concentrated in five KEGG pathways and five Panther pathways ${ }^{\text {a }}$.

\begin{tabular}{|c|c|c|c|}
\hline Term & P-value & Counts & Genes \\
\hline hsa05200:Pathways in cancer & $6.01 \times 10^{-04}$ & 20 & $\begin{array}{l}\text { CKS1B, AR, RET, E2F3, PDGFB, GNA12, } \\
\text { FOXO1, KITLG, FGF13, FGF12, CTNNA3, } \\
\text { RAD51, TPM3, EDNRB, FOS, WNT7B, } \\
\text { PAX8, TGFA, GNG4, PLCB1 }\end{array}$ \\
\hline hsa04720:Long-term potentiation & $1.27 \times 10^{-02}$ & 6 & $\begin{array}{l}\text { GRM5, RPS6KA6, GRIN2B, CAMK4, } \\
\text { RAPGEF3, PLCB1 }\end{array}$ \\
\hline hsa04014:Ras signaling pathway & $2.16 \times 10^{-02}$ & 11 & $\begin{array}{l}\text { LAT, GAB2, GRIN2B, RASGRF2, PDGFB, } \\
\text { EFNA3, KITLG, FGF13, FGF12, GNG4, } \\
\text { ABL2 }\end{array}$ \\
\hline hsa04080:Neuroactive ligand-receptor interaction & $3.31 \times 10^{-02}$ & 12 & $\begin{array}{l}\text { GRM5, EDNRB, GABRG3, PTGIR, } \\
\text { ADORA3, GRIN2B, P2RY4, CNR1, } \\
\text { ADRA1A, CHRNB2, HTR1D, GABRQ }\end{array}$ \\
\hline hsa04015:Rap1 signaling pathway & $3.42 \times 10^{-02}$ & 10 & $\begin{array}{l}\text { LAT, GRIN2B, PDGFB, CNR1, EFNA3, } \\
\text { KITLG, FGF13, FGF12, RAPGEF3, PLCB1 }\end{array}$ \\
\hline $\begin{array}{l}\text { P00026:Heterotrimeric G-protein signaling pathway-Gi } \\
\text { alpha and Gs alpha-mediated pathway }\end{array}$ & $2.26 \times 10^{-02}$ & 11 & $\begin{array}{l}\text { GRM5, GNAL, RET, ADORA3, FGB, RGS4, } \\
\text { RGS5, GPSM2, ADRA1A, HTR1D, PYGB }\end{array}$ \\
\hline P05730:Endogenous cannabinoid signaling & $3.57 \times 10^{-02}$ & 4 & GRM5, CNR1, PLCB1, GNG4 \\
\hline $\begin{array}{l}\text { P00027:Heterotrimeric G-protein signaling } \\
\text { pathway-Gq alpha and Go alpha-mediated pathway }\end{array}$ & $5.59 \times 10^{-02}$ & 9 & $\begin{array}{l}\text { GRM5, ADORA3, RGS4, RGS5, GPSM2, } \\
\text { RHOB, PLCB1, GNG4, RHOF }\end{array}$ \\
\hline $\begin{array}{l}\text { P05911:Angiotensin II-stimulated signaling through } \\
\text { G proteins and beta-arrestin }\end{array}$ & $5.81 \times 10^{-02}$ & 5 & EGR1, RHOB, PLCB1, GNG4, RHOF \\
\hline P00008:Axon guidance mediated by Slit/Robo & $6.51 \times 10^{-02}$ & 4 & ROBO1, RHOB, ABL2, RHOF \\
\hline
\end{tabular}

${ }^{\mathrm{a}} \mathrm{P}<0.05$. KEGG, Kyoto Encyclopedia of Genes and Genomes.

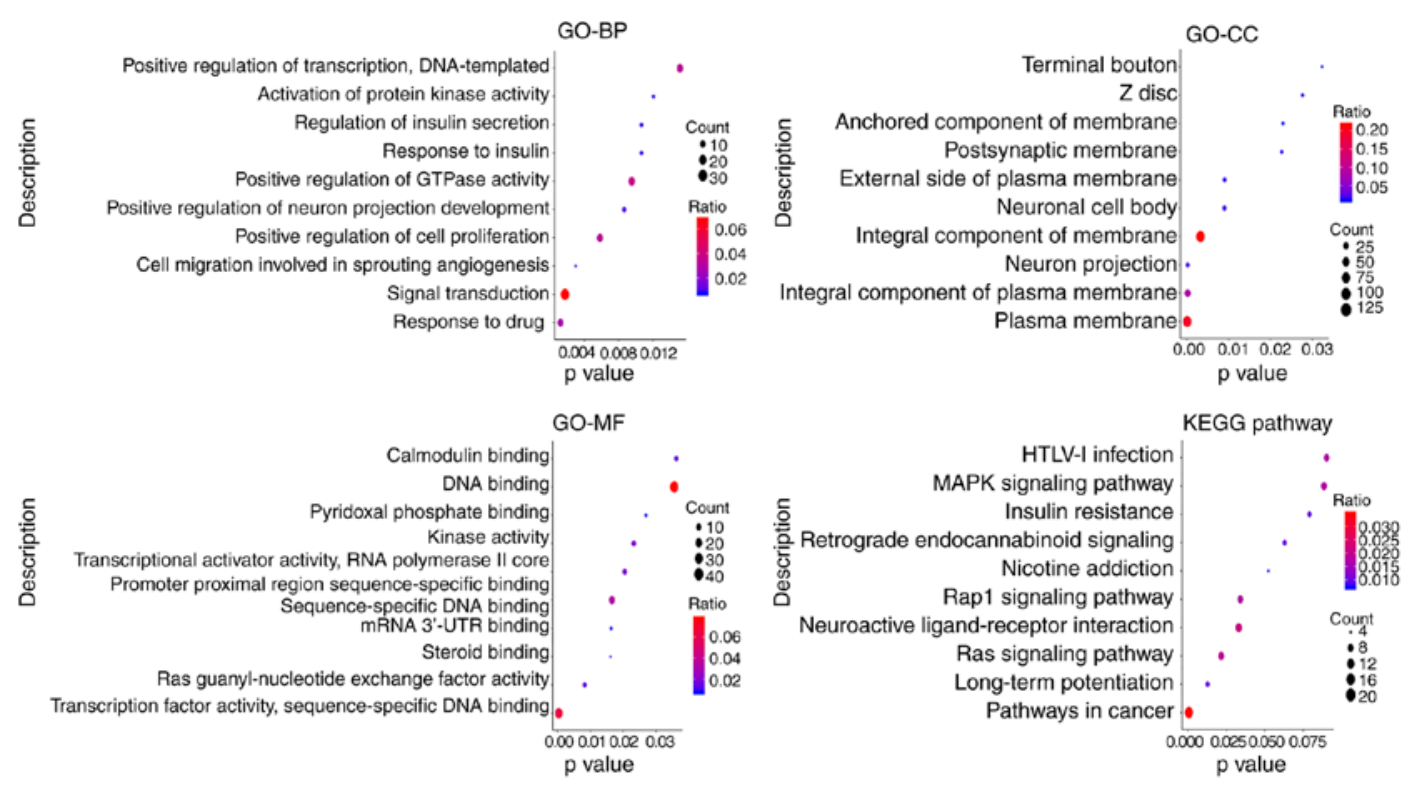

Figure 8. Bubble diagrams showing the results of the enrichment analysis and signaling pathway analysis of 382 hub genes regulated by miR-139-5p in HCC. $\mathrm{BP}$ of GO analysis; CC of GO analysis; MF of GO analysis; KEGG signaling pathways. HCC, hepatocellular carcinoma; GO, gene ontology; KEGG, Kyoto Encyclopedia of Genes and Genomes; bp, biological process; CC, cellular component; MF, molecular function; miR, microRNA.

signaling' were strongly enriched by predictive target genes. Importantly, miR-139-5p regulation serves an important role in tumor development and progression (63). Results from the present study revealed an association of miR-139-5p expression with progression and poor outcome in patients with HCC. Mitogenic signaling cascades associated with proliferation 
A

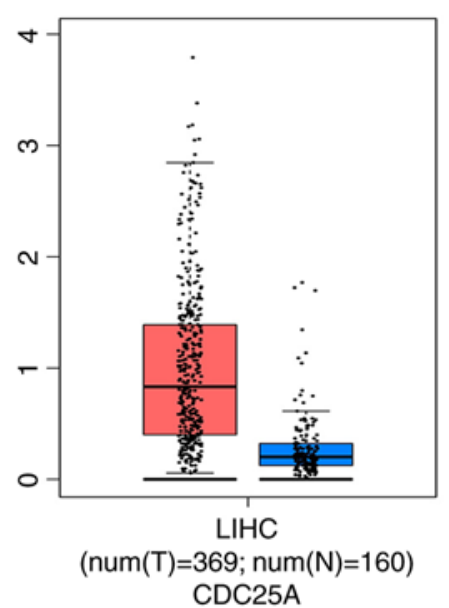

$\mathrm{D}$

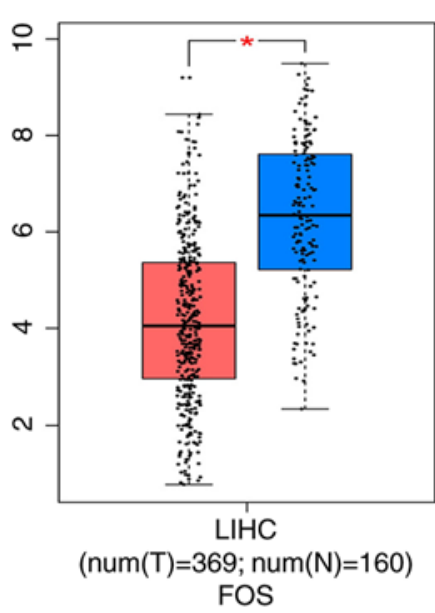

B

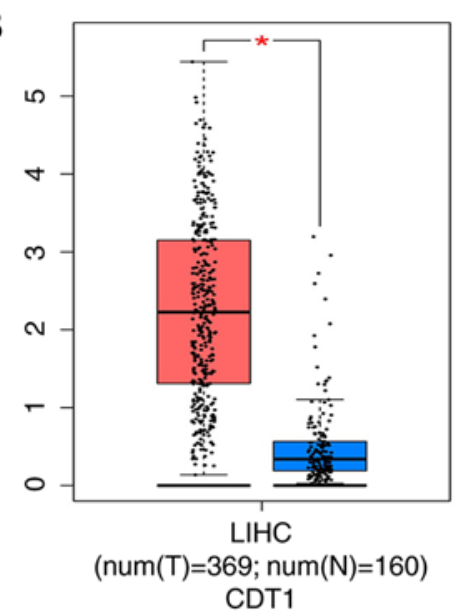

E

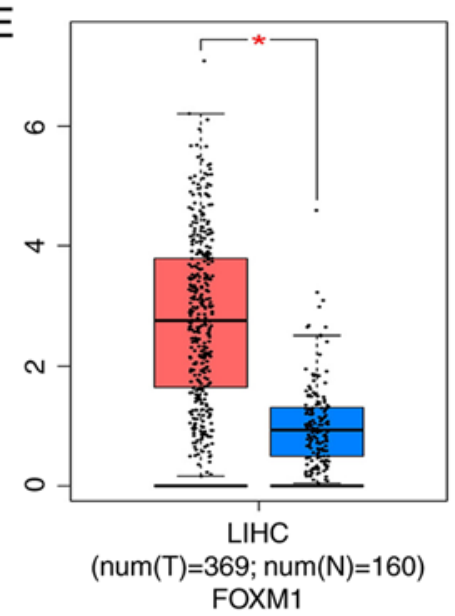

C

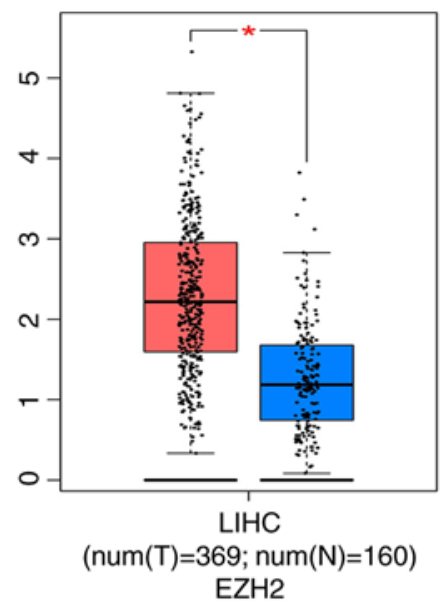

F

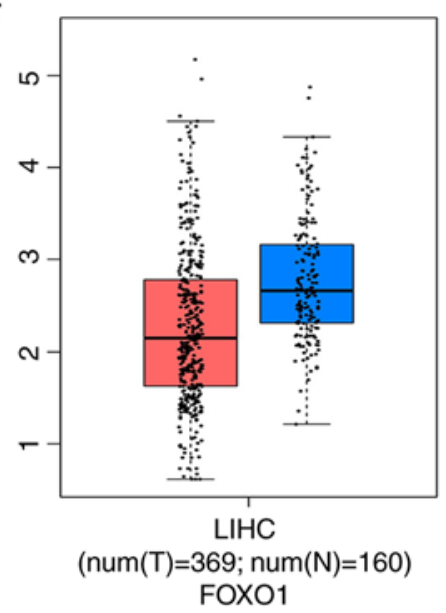

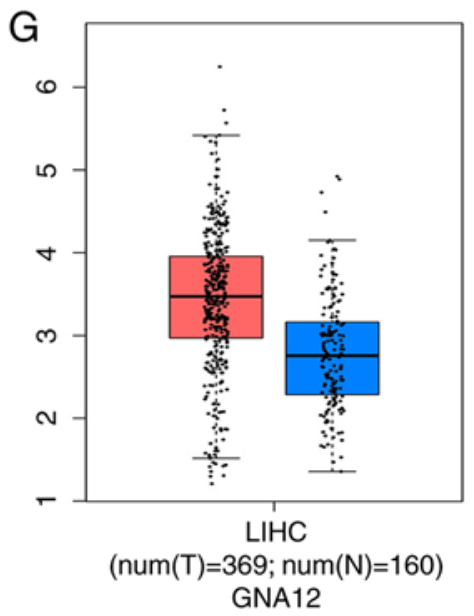

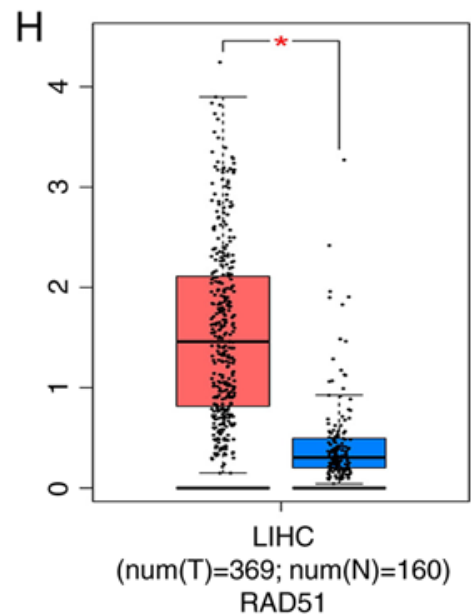

Figure 9. Expression of the top eight predicted target genes in HCC and nontumor liver tissues from TCGA. (A) CDC25A; (B) CDT1; (C) EZH2; (D) FOS; (E) FOXM1; (F) FOXO1; (G) GNA12; (H) RAD51. Significant upregulation of CDT1, EZH2, FOXM1 and RAD51 expression and downregulation of FOS in HCC tissues was observed ( $\mathrm{P}<0.05)$. No predominant differences were detected among the remaining genes, with $\mathrm{P}>0.05$. LIHC, Liver Hepatocellular Carcinoma; HCC, hepatocellular carcinoma; TCGA, The Cancer Genome Atlas; CDC25A, cell division cycle 25A; CDT1, chromatin licensing and DNA replication factor 1; EZH2, enhancer of zeste homolog 2; FOS, FBJ murine osteosarcoma viral oncogene homolog; FOXM1, forkhead box M1; FOXO1, forkhead box O1; GNA12, guanine nucleotide binding protein alpha 12; RAD51, recombination protein.

and translation are facilitated by activated Ras (63), and Ras regulates programmed cell death (64). Thus, an association exists between Ras alterations and carcinogenesis in various malignancies (65-67). Moreover, miRNAs have the ability to initiate the Ras-mitogen activated protein kinase pathway related to cell proliferation and survival, while no publication has reported an association between the Ras signaling pathway and miR-139 expression. Endogenous cannabinoid signaling, which is mainly determined by the activity of endocannabinoids $\mathrm{N}$-arachidonoylethanolamine and 2-arachidonoylglycerol, as well as cannabinoid receptor type receptors 1 and 2 has attracted increasing interest due 

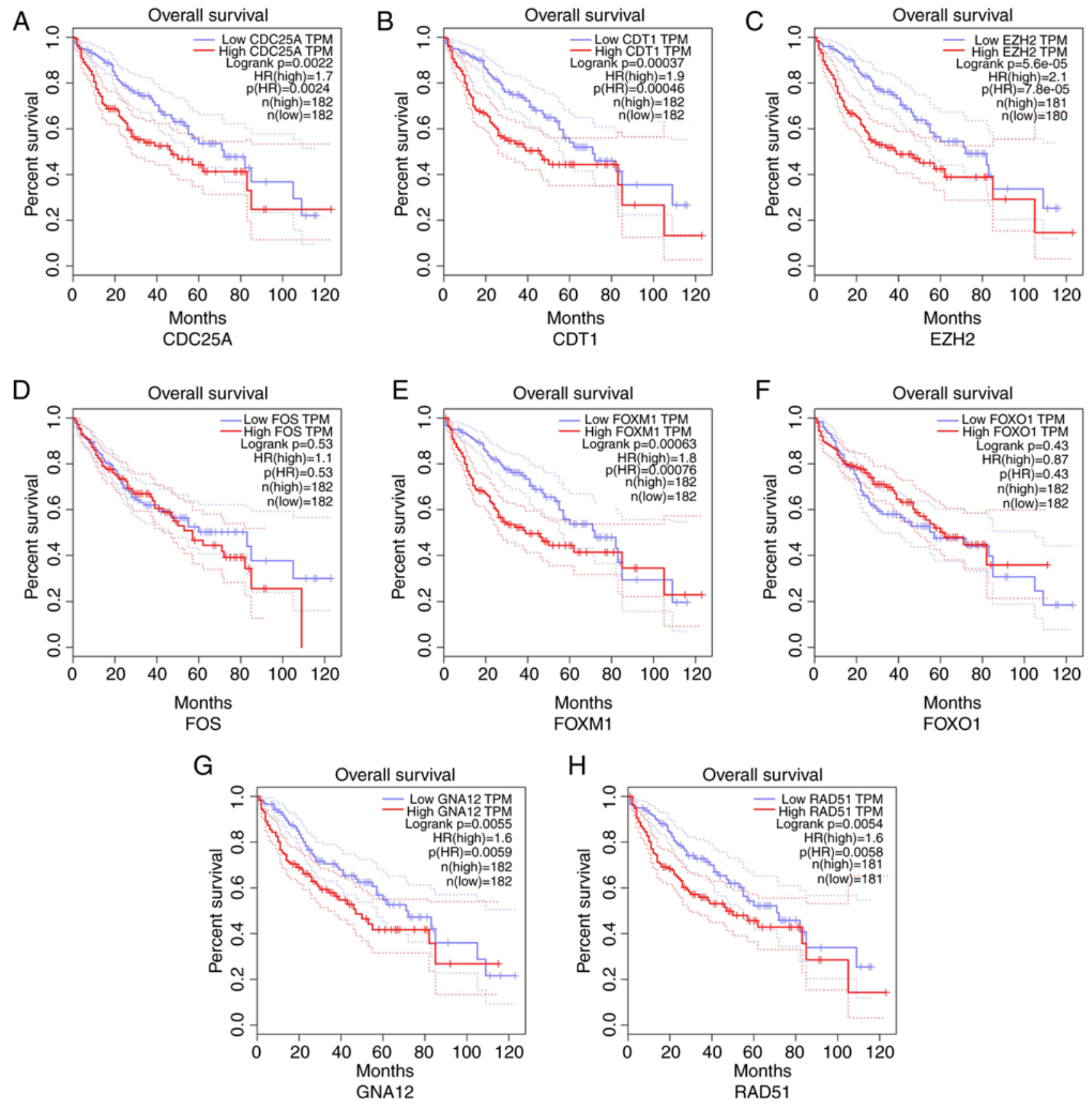

Figure 10. Kaplan-Meier curves displaying the associations between the expression of the top target genes and OS of patients with HCC. (A) CDC25A; (B) CDT1; (C) EZH2; (D) FOS; (E) FOXM1; (F) FOXO1; (G) GNA12; (H) RAD51. These results indicate that the high expression of CDT1, EZH2, RAD51, FOXM1, GNA12 and CDC25A had a significant effect on the OS of patients with $\mathrm{HCC}(\mathrm{P}<0.05)$ while no marked effect was detected in the expression of other genes in HCC ( $\mathrm{P}>0.05)$. HCC, hepatocellular carcinoma; OS, overall survival; CDC25A, cell division cycle 25A; CDT1, chromatin licensing and DNA replication factor 1; EZH2, enhancer of zeste homolog 2; FOS, FBJ murine osteosarcoma viral oncogene homolog; FOXM1, forkhead box M1; FOXO1, forkhead box O1; GNA12, guanine nucleotide binding protein alpha 12; RAD51, recombination protein; TPM, transcripts per million.

to its antitumor effects $(68,69)$. According to Vago et al $(70)$, the suppression of $\mathrm{N}$-acylethanolamine-hydrolyzing acid amidase activity regulates tumor cell death and migration, and controls the progression of bladder cancer, indicating a new therapeutic target for patients with bladder cancer. Furthermore, Martínez-Martínez et al (71) verified that activated CB2 accelerates the development of colon cancer via a mechanism dependent on the AKT/GSK3 $\beta$ signaling pathway. Nevertheless, a relationship between endogenous cannabinoid signaling and miR-139 regulation has not been experimentally confirmed, therefore further research is required.
To better understand the downstream mechanisms of the top eight target genes, biofunctional investigations of FOS, CDT1, EZH2, RAD51, FOXM1, FOXO1, GNA12 and CDC25A were conducted. CDT1, which is involved in the formation of the prereplication complex required for DNA fragment replication, functions as an oncogene in multiple tumor types, including HCC (72-75). Karavias et al (75) confirmed that increased CDT1 expression has a negative influence on the survival of patients with HCC and observed correlations between the upregulation of CDT1 and tumor grade and TNM stage by performing IHC staining in HCC tissues, supporting 

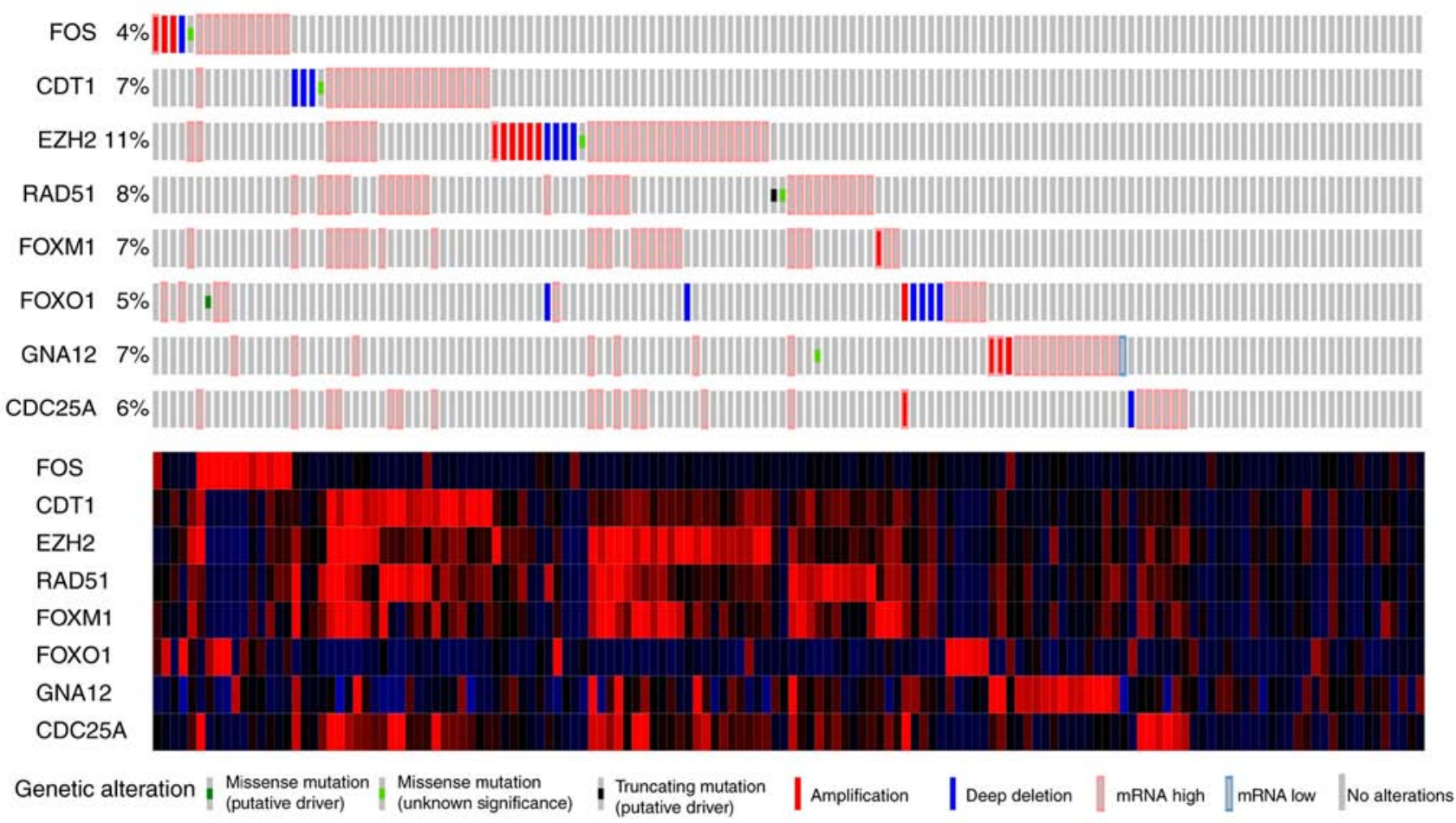

Figure 11. Frequency of mutations in the top eight target genes of 360 patients with HCC from the CBio Cancer Genomics Portal. HCC, hepatocellular carcinoma; CDC25A, cell division cycle 25A; CDT1, chromatin licensing and DNA replication factor 1; EZH2, enhancer of zeste homolog 2; FOS, FBJ murine osteosarcoma viral oncogene homolog; FOXM1, forkhead box M1; FOXO1, forkhead box O1; GNA12, guanine nucleotide binding protein alpha 12; RAD51, recombination protein.

the results of the present study (Figs. 9 and 11). Moreover, Yu et al (76) discovered a combination of new diagnostic indicators, including CDT1, MCM7, NUDT1, CENPM and HDAC11, with favorable diagnostic efficiency in HCC. Accordingly, CDT1 expression is crucial for the development and prognostic evaluation of HCC, thus further research is required to verify the association between miR-139-5p and CDT1 expression in HCC. RAD51 recombinase (RAD51), which participates in reestablishing and repairing homologous DNA, binds to the BRCA1 and BRCA2 genes, which are involved in tumorigenesis (77). Previous studies have reported that certain pharmaceutical molecules, including corylin, melatonin and gefitinib in combination with irinotecan, were able to enhance cancer cell sensitivity to chemotherapy by inhibiting RAD51-induced DNA repair in HCC (78-80). Luo et al (81) observed increased expression of RAD51 in HCC tissues, consistent with the findings of the present study, and revealed that miRNA-146a-5p enhanced the radio-sensitivity of HCC cells via the DNA repair pathway. However, studies have not yet reported an association between miR-139-5p and RAD51 regulation in patients with HCC. FOXM1 and FOXO1, members of the forkhead family, serve vital roles in cell proliferation and gene transcription. According to certain studies (82-85), FOXM1 overexpression results in poor survival and undesirable development of HCC, consistent with the present study results (Figs. 9 and 10). Lin et al (86) confirmed the reduced expression of FOXO1 in HCC tissues using IHC, and reported that FOXO1 served an important role in the development of HCC by regulating miRNA expression (87-89). CDC25A, as a member of the CDC25 family of phosphatases, mediates the $\mathrm{G} 1$ to $\mathrm{S}$ phase transition. A strong correlation between $\mathrm{CDC} 25$ expression and poor prognosis of patients with HCC was demonstrated by $\mathrm{Xu}$ et al (90) and a relevant study reported that the suppression of CDC25 expression halts the proliferation and progression of HCC (91). GNA12 has been found to possess carcinogenic potential and accelerate the progression of certain tumors (92-95). No studies have focused on the relevancy between miR-139-5p expression and FOXM1, FOXO1, GNA12 and CDC25A in HCC. The present study may provide novel insights for subsequent studies. FOS has multiple functions in cell proliferation, differentiation, transformation and apoptosis. Moreover, an association between FOS expression and HCC development has been reported (96-98). Fan et al (98) indicated that decreased miRNA-139 expression potentially facilitates the progression and metastasis of HCC via inhibiting FOS expression. Further research is necessary.

In short, it should be noted that miR-139-5p expression has a strong impact on the progression and prognosis of HCC mainly via targeted regulation of EZH2 and FOS expression. Moreover, the results of the present study clearly demonstrated that the regulation of FOS and FOXO1 was positively associated with miR-139-5p expressed in HCC. However, GNA12 and RAD51 expression levels were negatively associated with miR-139-5p expression based on the TCGA data. However, no studies have yet demonstrated the involvement of miR-139-5p in HCC development via targeted modulation of CDT1, RAD51, FOXM1, FOXO1, GNA12 and CDC25A expression.

The present study had certain limitations. Firstly, only four publications described a relationship among miR-139-5p regulation and prognostic effect size, including OS, DFS and RFS in HCC. Secondly, the HRs and corresponding 95\% CIs were directly obtained from Kaplan-Meier curves, which 
FOS

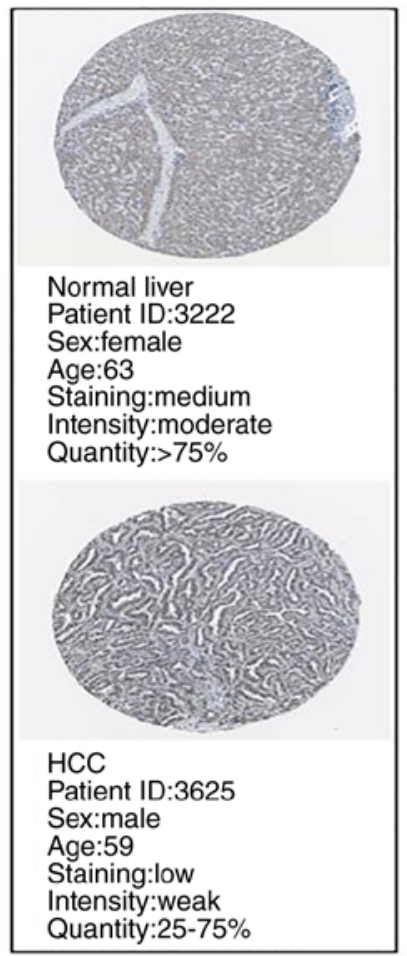

FOXO1

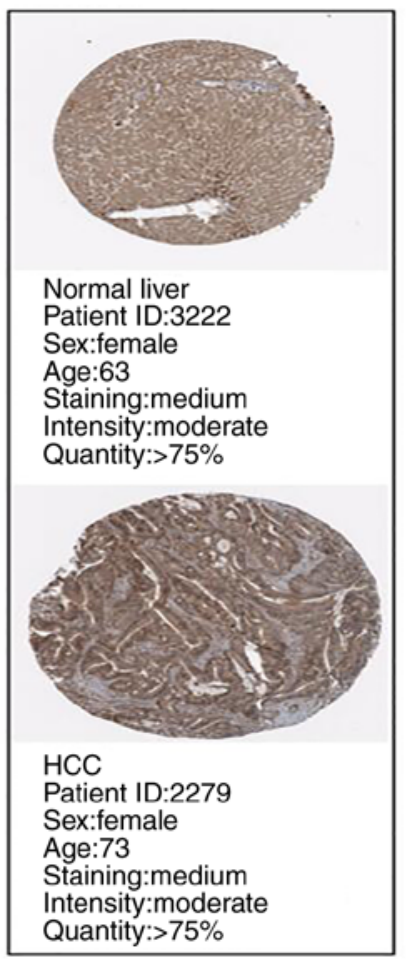

GNA12

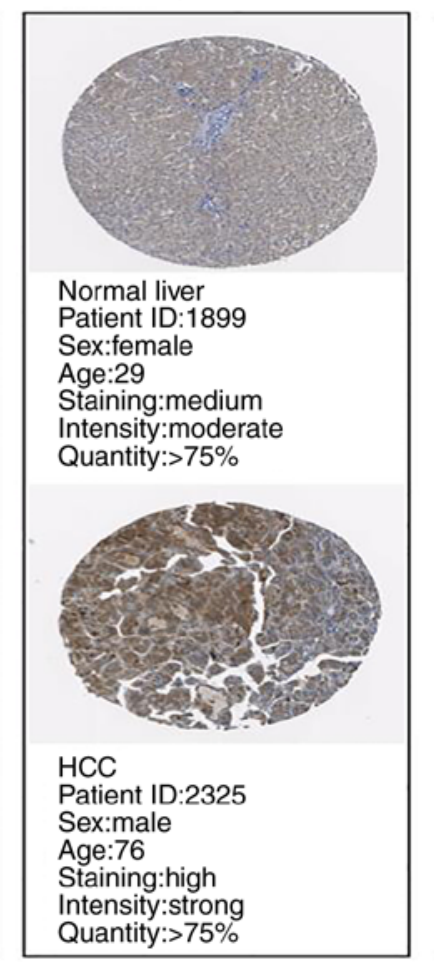

RAD51

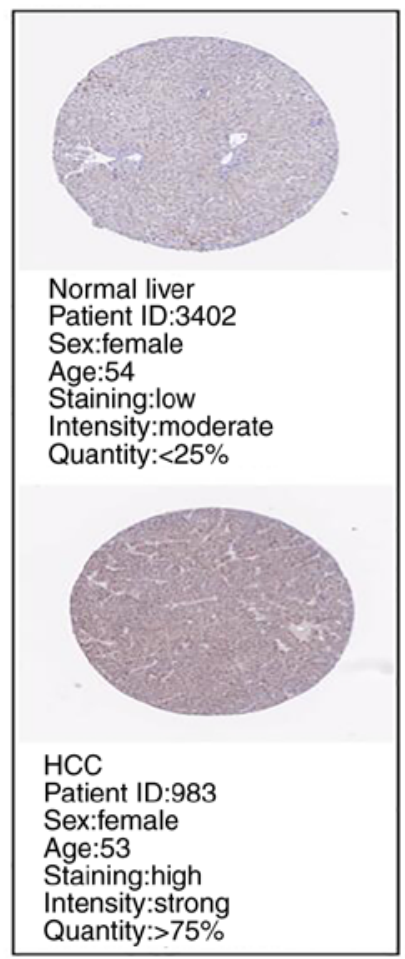

Figure 12. Verification of the levels of the proteins encoded by the four hub genes in normal and HCC tissues using the Human Protein Atlas database. HCC, hepatocellular carcinoma; FOS, FBJ murine osteosarcoma viral oncogene homolog; FOXO1, forkhead box O1; GNA12, guanine nucleotide binding protein alpha 12; RAD51, recombination protein.
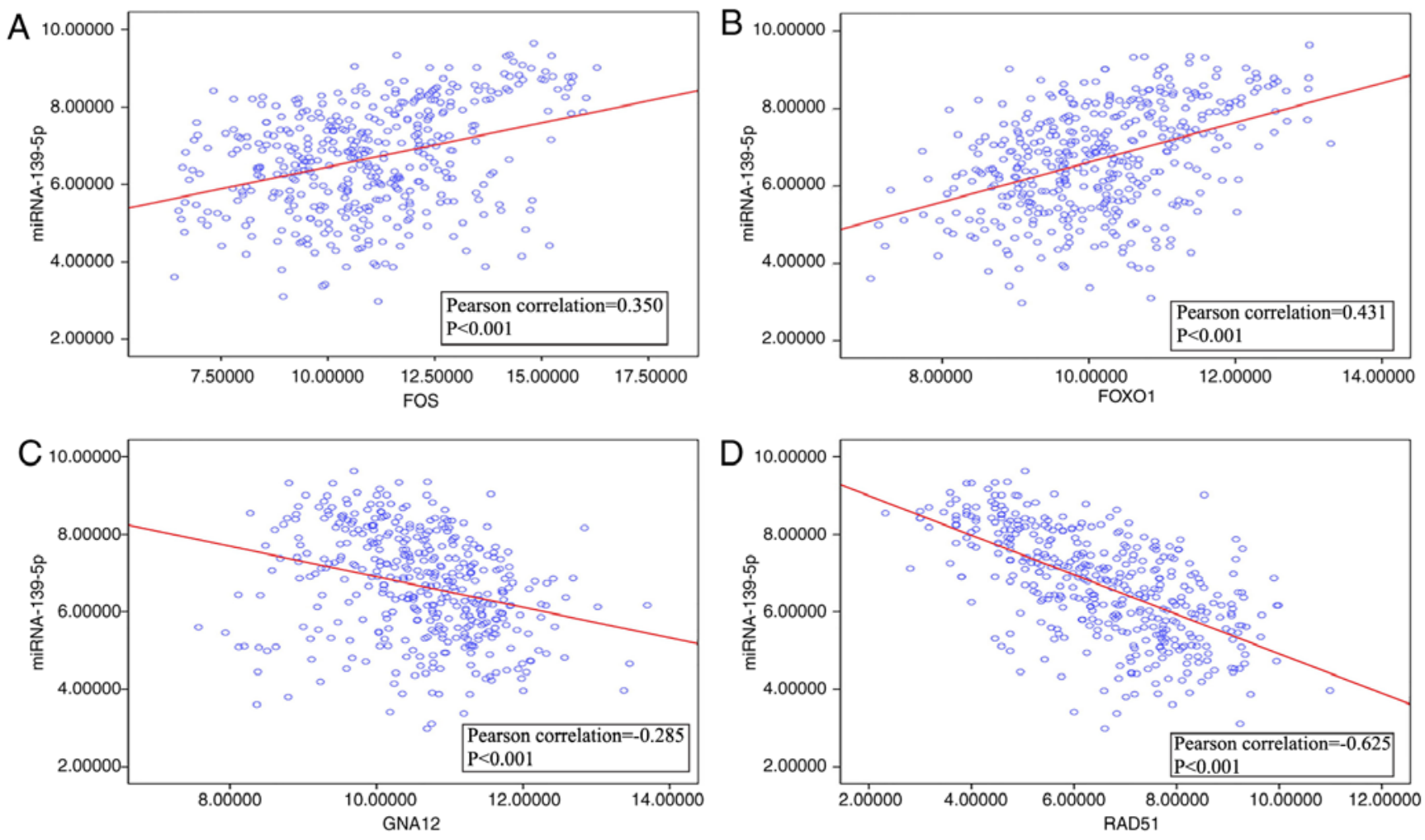

Figure 13. Evaluation of the correlation between the expression of the four hub genes and miR-139-5p regulation in patients with hepatocellular carcinoma from The Cancer Genome Atlas. (A) A positive correlation between miR-139-5p and FOS regulation was detected, with a Pearson correlation coefficient of 0.350 and $\mathrm{P}<0.001$. (B) A positive correlation between miR-139-5p and FOXO1 regulation was identified, with a Pearson correlation coefficient of 0.431 and $\mathrm{P}<0.001$. (C) A negative correlation between miR-139-5p and GNA12 regulation was detected, with a Pearson correlation coefficient of -0.285 and $\mathrm{P}<0.001$ (D) A negative correlation between miR-139-5p and RAD51 regulation was observed, with a Pearson correlation coefficient of -0.625 and P<0.001. FOS, FBJ murine osteosarcoma viral oncogene homolog; FOXO1, forkhead box O1; GNA12, guanine nucleotide binding protein alpha 12; RAD51, recombination protein; miR, microRNA. 
generated biased outcomes due to limited data. Thirdly, the included miRNA-seq and microarray data were obtained from diverse platforms and channels, suggesting clear heterogeneity and poor diagnostic efficiency. Accordingly, a uniform method for detecting miR-139-5p expression in HCC should be formulated. Moreover, evidence for signaling pathways that are regulated by miR-139-5p via the targeted modulation of hub gene expression is lacking, and further in vitro and in vivo studies are required to determine the precise biological effects of the target genes on HCC development.

In summary, the findings of the present study revealed that miR-139-5p expression was lower in HCC tissues than in nontumorous tissues and strong associations were observed for miR-139-5p expression with tumor grade, pathological stage and T stage. Additionally, reduced miR-139-5p expression generally led to an undesirable prognosis in patients with HCC. Moreover, the top eight target genes, namely FOS, CDT1, EZH2, RAD51, FOXM1, FOXO1, GNA12 and CDC25A induced by miR-139-5p expression were revealed to be involved in several pathways, including 'response to drug', 'pathways in cancer', 'Ras signaling pathway' and 'endogenous cannabinoid signaling'. These genes may have a major impact on carcinogenesis and the development of HCC. The current study provided a comprehensive investigation of the role of miR-139-5p in HCC progression. Nevertheless, additional research of the underlying molecular mechanisms related to $\mathrm{HCC}$, including in vitro and in vivo experiments, should be further conducted to confirm the outcomes of the present study.

\section{Acknowledgements}

Not applicable.

\section{Funding}

The present study was supported by the Guangxi Science and Technology Program (grant no. GuiKeAB17195020) and the National Natural Science Foundation of China (grant no. NSFC81860319).

\section{Availability of data and materials}

The datasets used and/or analyzed during the present study are available from the corresponding author on reasonable request.

\section{Authors' contributions}

HQ, BML, XDW, DYW, HY and YH made substantial contributions to the conception and design of the present study. HQ, QQ, YTP and CYZ assisted in the acquisition, analysis and interpretation of data. HQ, DYW, BML, YH and HY were involved in drafting the manuscript and BML, $\mathrm{YH}$ and $\mathrm{HY}$ critically revising it for important intellectual content. All authors gave final approval for the version of the manuscript to be published.

\section{Ethics approval and consent to participate}

Not applicable.

\section{Patient consent for publication}

Not applicable.

\section{Competing interests}

The authors declare that they have no competing interests.

\section{References}

1. Bray F and Soerjomataram I: The changing global burden of cancer: Transitions in human development and implications for cancer prevention and control.

2. Bray F, Ferlay J, Soerjomataram I, Siegel RL, Torre LA and Jemal A: Global cancer statistics 2018: GLOBOCAN estimates of incidence and mortality worldwide for 36 cancers in 185 countries. CA Cancer J Clin 68: 394-424, 2018.

3. Siegel RL, Miller KD and Jemal A: Cancer statistics, 2017. CA Cancer J Clin 67: 7-30, 2017.

4. Yeh SH and Chen PJ: Gender disparity of hepatocellular carcinoma: The roles of sex hormones. Oncology (78 Suppl 1): S172-S179, 2010

5. Aghemo A: Update on HCC management and review of the new EASL guidelines. Gastroenterol Hepatol (NY) 14: 384-386, 2018.

6. Singal AG and El-Serag HB: Hepatocellular carcinoma from epidemiology to prevention: Translating knowledge into practice. Clin Gastroenterol Hepatol 13: 2140-2151, 2015.

7. Otedo A, Simbiri KO, Were V, Ongati O and Estambale BA: Risk factors for liver Cancer in HIV endemic areas of Western Kenya. Infect Agent Cancer 13: 41, 2018.

8. Oweira H, Petrausch U, Helbling D, Schmidt J, Mehrabi A, Schöb O, Giryes A and Abdel-Rahman O: Prognostic value of site-specific extra-hepatic disease in hepatocellular carcinoma: A SEER database analysis. Expert Rev Gastroenterol Hepatol 11: 695-701, 2017.

9. Intaraprasong P, Siramolpiwat S and Vilaichone RK: Advances in management of hepatocellular carcinoma. Asian Pac J Cancer Prev 17: 3697-3703, 2016.

10. Valinezhad Orang A, Safaralizadeh R and Kazemzadeh-Bavili M: Mechanisms of miRNA-mediated gene regulation from common downregulation to mRNA-specific upregulation. Int J Genomics 2014: 970607, 2014.

11. Fanini F and Fabbri M: MicroRNAs and cancer resistance: A new molecular plot. Clin Pharmacol Ther 99: 485-493, 2016.

12. Bartel DP: MicroRNAs: Target recognition and regulatory functions. Cell 136: 215-233, 2009.

13. Mitchell PS, Parkin RK, Kroh EM, Fritz BR, Wyman SK, Pogosova-Agadjanyan EL, Peterson A, Noteboom J, O'Briant KC, Allen A, et al: Circulating microRNAs as stable blood-based markers for cancer detection. Proc Natl Acad Sci USA 105: 10513-10518, 2008.

14. Manikandan J, Aarthi JJ, Kumar SD and Pushparaj PN: Oncomirs: The potential role of non-coding microRNAs in understanding cancer. Bioinformation 2: 330-334, 2008.

15. Yates LA, Norbury CJ and Gilbert RJ: The long and short of microRNA. Cell 153: 516-519, 2013.

16. Beca F and Schmitt F: MicroRNA signatures in cytopathology: Are they ready for prime time? Cancer Cytopathol 124: 613-615, 2016.

17. Wang J, Chen J and Sen S: MicroRNA as biomarkers and diagnostics. J Cell Physiol 231: 25-30, 2016.

18. Keller A, Rounge T, Backes C, Ludwig N, Gislefoss R, Leidinger P, Langseth $\mathrm{H}$ and Meese E: Sources to variability in circulating human miRNA signatures. RNA Biol 14: 1791-1798, 2017.

19. Chen Z, Yu T, Cabay RJ, Jin Y, Mahjabeen I, Luan X, Huang L, Dai Y and Zhou X: miR-486-3p, miR-139-5p, and miR-21 as biomarkers for the detection of oral tongue squamous cell carcinoma. Biomark Cancer 9: 1-8, 2017.

20. Zou F, Mao R, Yang L, Lin S, Lei K, Zheng Y, Ding Y, Zhang P, Cai G, Liang $X$ and Liu J: Targeted deletion of miR-139-5p activates MAPK, NF- $\kappa \mathrm{B}$ and STAT3 signaling and promotes intestinal inflammation and colorectal cancer. FEBS J 283: 1438-1452, 2016.

21. Liu H, Yin Y, Hu Y, Feng Y, Bian Z, Yao S, Li M, You Q and Huang Z: miR-139-5p sensitizes colorectal cancer cells to 5-fluorouracil by targeting NOTCH-1. Pathol Res Pract 212: 643-649, 2016. 
22. Hu Y, Deng C, Zhang H, Zhang J, Peng B and Hu C: Long non-coding RNA XIST promotes cell growth and metastasis through regulating miR-139-5p mediated Wnt/ $\beta$-catenin signaling pathway in bladder cancer. Oncotarget 8: 94554-94568, 2017.

23. Liu J, Li C, Jiang Y, Wan Y, Zhou S and Cheng W: Tumor-suppressor role of miR-139-5p in endometrial cancer. Cancer Cell Int 18: 51, 2018.

24. Hua S, Lei L, Deng L, Weng X, Liu C, Qi X, Wang S, Zhang D, Zou X, Cao C, et al: miR-139-5p inhibits aerobic glycolysis, cell proliferation, migration, and invasion in hepatocellular carcinoma via a reciprocal regulatory interaction with ETS1. Oncogene 37: 1624-1636, 2018.

25. Wang Z, Ding Q, Li Y, Liu Q, Wu W, Wu L and Yu H: Reanalysis of microRNA expression profiles identifies novel biomarkers for hepatocellular carcinoma prognosis. Tumour Biol 37: 14779-14787, 2016.

26. Ni H, Dai X, Leng X, Deng M, Qin Y, Ji Q, Xu C, Li J and Liu Y: Higher variety and quantity of microRNA-139-5p isoforms confer suppressive role in hepatocellular carcinoma. J Cell Biochem 119: 6806-6813, 2018.

27. Wang YH, Ji J, Weng H, Wang BC and Wang FB: MiR-139 in digestive system tumor diagnosis and detection: Bioinformatics and meta-analysis. Clin Chim Acta 485: 33-41, 2018.

28. Wang L, Liu M, Zhu H, Rong W, Wu F, An S, Liu F, Feng L, Wu J and Xu N: Identification of recurrence-related serum microRNAs in hepatocellular carcinoma following hepatectomy. Cancer Biol Ther 16: 1445-1452, 2015.

29. Wong CC, Wong CM, Tung EK, Au SL, Lee JM, Poon RT, Man K and Ng IO: The microRNA miR-139 suppresses metastasis and progression of hepatocellular carcinoma by down-regulating Rho-kinase 2. Gastroenterology 140 : 322-331, 2011

30. Clough $\mathrm{E}$ and Barrett $\mathrm{T}$ : The gene expression omnibus database. Methods Mol Biol 1418: 93-110, 2016.

31. Tomczak K, Czerwińska P and Wiznerowicz M: The Cancer Genome Atlas (TCGA): An immeasurable source of knowledge. Contemp Oncol (Pozn) 19: A68-A77, 2015.

32. Parkinson H, Sarkans U, Kolesnikov N, Abeygunawardena N, Burdett T, Dylag M, Emam I, Farne A, Hastings E, Holloway E, et al: ArrayExpress update-an archive of microarray and high-throughput sequencing-based functional genomics experiments. Nucleic Acids Res 39: D1002-D1004, 2011.

33. Rhodes DR, Yu J, Shanker K, Deshpande N, Varambally R, Ghosh D, Barrette T, Pandey A and Chinnaiyan AM: ONCOMINE: A cancer microarray database and integrated data-mining platform. Neoplasia 6: 1-6, 2004.

34. Tierney JF, Stewart LA, Ghersi D, Burdett S and Sydes MR: Practical methods for incorporating summary time-to-event data into meta-analysis. Trials 8: 16, 2007.

35. Vamvakas EC: Meta-analyses of studies of the diagnostic accuracy of laboratory tests: A review of the concepts and methods. Arch Pathol Lab Med 122: 675-686, 1998.

36. Sticht C, De La Torre C, Parveen A and Gretz N: miRWalk: An online resource for prediction of microRNA binding sites. PLoS One 13: e0206239, 2018.

37. Chou CH, Shrestha S, Yang CD, Chang NW, Lin YL, Liao KW, Huang WC, Sun TH, Tu SJ, Lee WH, et al: miRTarBase update 2018: A resource for experimentally validated microRNA-target interactions. Nucleic Acids Res 46: D296-D302, 2018.

38. Vergoulis T, Vlachos IS, Alexiou P, Georgakilas G Maragkakis M, Reczko M, Gerangelos S, Koziris N, Dalamagas T and Hatzigeorgiou AG: TarBase 6.0: Capturing the exponential growth of miRNA targets with experimental support. Nucleic Acids Res 40: D222-D229, 2012.

39. Bandyopadhyay S and Mitra R: TargetMiner: MicroRNA target prediction with systematic identification of tissue-specific negative examples. Bioinformatics 25: 2625-2631, 2009.

40. Bhattacharya A, Ziebarth JD and Cui Y: PolymiRTS Database 3.0: Linking polymorphisms in microRNAs and their target sites with human diseases and biological pathways. Nucleic Acids Res 42: D86-D91, 2014.

41. Loher P and Rigoutsos I: Interactive exploration of RNA22 microRNA target predictions. Bioinformatics 28: 3322-3323, 2012.

42. Betel D, Wilson M, Gabow A, Marks DS and Sander C: The microRNA.org resource: Targets and expression. Nucleic Acids Res 36: D149-D153, 2008.

43. John B, Enright AJ, Aravin A, Tuschl T, Sander C and Marks DS: Human MicroRNA targets. PLoS Biol 2: e363, 2004.
44. Hsu SD, Chu CH, Tsou AP, Chen SJ, Chen HC, Hsu PW, Wong YH, Chen YH, Chen GH and Huang HD: miRNAMap 2.0: Genomic maps of microRNAs in metazoan genomes. Nucleic Acids Res 36: D165-D169, 2008.

45. Agarwal V, Bell GW, Nam JW and Bartel DP: Predicting effective microRNA target sites in mammalian mRNAs. Elife 4, 2015

46. Liu W and Wang X: Prediction of functional microRNA targets by integrative modeling of microRNA binding and target expression data. Genome Biol 20: 18, 2019.

47. Krek A, Grun D, Poy MN, Wolf R, Rosenberg L, Epstein EJ, MacMenamin P, da Piedade I, Gunsalus KC, Stoffel M and Rajewsky N: Combinatorial microRNA target predictions. Nat Genet 37: 495-500, 2005.

48. Dennis G Jr, Sherman BT, Hosack DA, Yang J, Gao W, Lane HC and Lempicki RA: DAVID: Database for annotation, visualization, and integrated discovery. Genome Biol 4: P3, 2003.

49. Szklarczyk D, Franceschini A, Kuhn M, Simonovic M, Roth A, Minguez P, Doerks T, Stark M, Muller J, Bork P, et al: The STRING database in 2011: Functional interaction networks of proteins, globally integrated and scored. Nucleic Acids Res 39: D561-D568, 2011.

50. Szklarczyk D, Morris JH, Cook H, Kuhn M, Wyder S, Simonovic M, Santos A, Doncheva NT, Roth A, Bork P, et al: The STRING database in 2017: Quality-controlled protein-protein association networks, made broadly accessible. Nucleic Acids Res 45: D362-D368, 2017.

51. Tang Z, Li C, Kang B, Gao G, Li C and Zhang Z: GEPIA: A web server for cancer and normal gene expression profiling and interactive analyses. Nucleic Acids Res 45: W98-W102, 2017.

52. Pontén F, Schwenk JM, Asplund A and Edqvist PH: The human protein atlas as a proteomic resource for biomarker discovery. J Intern Med 270: 428-446, 2011.

53. Cerami E, Gao J, Dogrusoz U, Gross BE, Sumer SO, Aksoy BA, Jacobsen A, Byrne CJ, Heuer ML, Larsson E, et al: The cBio cancer genomics portal: An open platform for exploring multidimensional cancer genomics data. Cancer Discov 2: 401-404, 2012.

54. Li T, Yin J, Yuan L, Wang S, Yang L, Du X and Lu J: Downregulation of microRNA-139 is associated with hepatocellular carcinoma risk and short-term survival. Oncol Rep 31: 1699-1706, 2014

55. Jiao W, Zhang J, Wei Y, Feng J, Ma M, Zhao H, Wang L and Jiao W: MiR-139-5p regulates VEGFR and downstream signaling pathways to inhibit the development of esophageal cancer. Dig Liver Dis 51: 149-156, 2019.

56. Qin L, Deng HY, Chen SJ, Wei W and Zhang YT: miR-139 acts as a tumor suppressor in T-cell acute lymphoblastic leukemia by targeting CX chemokine receptor 4 . Am J Transl Res 9: 4059-4070, 2017

57. Chen J, Yu Y, Chen X, He Y, Hu Q, Li H, Han Q, Ren F, Li J, $\mathrm{Li} \mathrm{C}$, et al: MiR-139-5p is associated with poor prognosis and regulates glycolysis by repressing PKM2 in gallbladder carcinoma. Cell Prolif 51: e12510, 2018.

58. Zhang HD, Sun DW, Mao L, Zhang J, Jiang LH, Li J, Wu Y, Ji H, Chen W, Wang J, et al: MiR-139-5p inhibits the biological function of breast cancer cells by targeting Notch1 and mediates chemosensitivity to docetaxel. Biochem Biophys Res Commun 465: 702-713, 2015.

59. Ren Y, Zhu H, Chi C, Yang F and Xu X: MiRNA-139 regulates oral cancer Tca8113 cells apoptosis through Akt signaling pathway. Int J Clin Exp Pathol 8: 4588-4594, 2015.

60. Au SL, Wong CC, Lee JM, Fan DN, Tsang FH, Ng IO and Wong CM: Enhancer of zeste homolog 2 epigenetically silences multiple tumor suppressor microRNAs to promote liver cancer metastasis. Hepatology 56: 622-631, 2012.

61. Xu K, Shen K, Liang X, Li Y, Nagao N, Li J, Liu J and Yin P: MiR-139-5p reverses CD44+/CD133+-associated multidrug resistance by downregulating NOTCH1 in colorectal carcinoma cells. Oncotarget 7: 75118-75129, 2016.

62. Yoon EL, Yeon JE, Ko E, Lee HJ, Je JH, Yoo YJ, Kang SH, Suh SJ, Kim JH, Seo YS, et al: An explorative analysis for the role of serum miR-10b-3p levels in predicting response to sorafenib in patients with advanced hepatocellular carcinoma. J Korean Med Sci 32: 212-220, 2017.

63. Huang LL, Huang LW, Wang L, Tong BD, Wei Q and Ding XS: Potential role of miR-139-5p in cancer diagnosis, prognosis and therapy. Oncol Lett 14: 1215-1222, 2017.

64. Cox AD and Der CJ: The dark side of Ras: Regulation of apoptosis. Oncogene 22: 8999-9006, 2003.

65. Bos JL: Ras oncogenes in human cancer: A review. Cancer Res 49: 4682-4689, 1989. 
66. Bos JL: The ras gene family and human carcinogenesis. Mutat Res 195: 255-271, 1988.

67. Prior IA, Lewis PD and Mattos C: A comprehensive survey of Ras mutations in cancer. Cancer Res 72: 2457-2467, 2012.

68. Schwarz R, Ramer R and Hinz B: Targeting the endocannabinoid system as a potential anticancer approach. Drug Metab Rev 50: 26-53, 2018.

69. Lu Y and Anderson HD: Cannabinoid signaling in health and disease. Can J Physiol Pharmacol 95: 311-327, 2017.

70. Vago R, Bettiga A, Salonia A, Ciuffreda $P$ and Ottria R: Development of new inhibitors for $\mathrm{N}$-acylethanolamine-hydrolyzing acid amidase as promising too against bladder cancer. Bioorg Med Chem 25: 1242-1249, 2017.

71. Martínez-Martínez E, Martín-Ruiz A, Martín P, Calvo V, Provencio $M$ and García JM: CB2 cannabinoid receptor activation promotes colon cancer progression via AKT/GSK3 $\beta$ signaling pathway. Oncotarget 7: 68781-68791, 2016.

72. Liontos M, Koutsami M, Sideridou M, Evangelou K, Kletsas D, Levy B, Kotsinas A, Nahum O, Zoumpourlis V, Kouloukoussa M, et al: Deregulated overexpression of hCdt1 and hCdc6 promotes malignant behavior. Cancer Res 67: 10899-10909, 2007.

73. Bravou V, Nishitani H, Song SY, Taraviras S and Varakis J: Expression of the licensing factors, Cdt1 and Geminin, in human colon cancer. Int J Oncol 27: 1511-1518, 2005.

74. Arentson E, Faloon P, Seo J, Moon E, Studts JM, Fremont DH and Choi K: Oncogenic potential of the DNA replication licensing protein CDT1. Oncogene 21: 1150-1158, 2002.

75. Karavias D, Maroulis I, Papadaki H, Gogos C, Kakkos S, Karavias D and Bravou V: Overexpression of CDT1 is a predictor of poor survival in patients with hepatocellular carcinoma. J Gastrointest Surg 20: 568-579, 2016.

76. Yu Z, Wang R, Chen F, Wang J and Huang X: Five novel oncogenic signatures could be utilized as AFP-related diagnostic biomarkers for hepatocellular carcinoma based on next-generation sequencing. Dig Dis Sci 63: 945-957, 2018.

77. Zhong Q, Chen CF, Li S, Chen Y, Wang CC, Xiao J, Chen PL, Sharp ZD and Lee WH: Association of BRCA1 with the hRad50-hMre11-p95 complex and the DNA damage response. Science 285: 747-750, 1999

78. Chen CC, Chen CY, Ueng SH, Hsueh C, Yeh CT, Ho JY, Chou LF and Wang TH: Corylin increases the sensitivity of hepatocellular carcinoma cells to chemotherapy through long noncoding RNA RAD51-AS1-mediated inhibition of DNA repair. Cell Death Dis 9: 543, 2018.

79. Chen CC, Chen CY, Wang SH, Yeh CT, Su SC, Ueng SH, Chuang WY, Hsueh C and Wang TH: Melatonin sensitizes hepatocellular carcinoma cells to chemotherapy through long non-coding RNA RAD51-AS1-mediated suppression of DNA repair. Cancers (Basel) 10, 2018.

80. Shao J, Xu Z, Peng X, Chen M, Zhu Y, Xu L, Zhu H, Yang B, Luo $P$ and He Q: Gefitinib synergizes with irinotecan to suppress hepatocellular carcinoma via antagonizing Rad51-mediated DNA-repair. PLoS One 11: e0146968, 2016.

81. Luo J, Si ZZ, Li T, Li JQ, Zhang ZQ, Chen GS, Qi HZ and Yao HL: MicroRNA-146a-5p enhances radiosensitivity in hepatocellular carcinoma through replication protein A3-induced activation of the DNA repair pathway. Am J Physiol Cell Physiol 316 : C299-C311, 2019.

82. Chand V, Pandey A, Kopanja D, Guzman G and Raychaudhuri P: Opposing roles of the forkhead box factors FoxM1 and FoxA2 in liver cancer. Mol Cancer Res 17: 1063-1074, 2019.

83. Liang C, Zhao J, Ge H, Li G and Wu J: Clinicopathological and prognostic significance of FoxM1 in hepatocellular carcinoma patients: A meta-analysis. Onco Targets Ther 11: 3561-3571, 2018.
84. Tian C, Wu H, Li C, Tian X, Sun Y, Liu E, Liao X and Song W: Downreguation of FoxM1 by miR-214 inhibits proliferation and migration in hepatocellular carcinoma. Gene Ther 25: 312-319, 2018.

85. Song BN and Chu IS: A gene expression signature of FOXM1 predicts the prognosis of hepatocellular carcinoma. Exp Mol Med 50: e418, 2018

86. Lin P, He RQ, Dang YW, Wen DY, Ma J, He Y, Chen G and Yang $\mathrm{H}$ : An autophagy-related gene expression signature for survival prediction in multiple cohorts of hepatocellular carcinoma patients. Oncotarget 9: 17368-17395, 2018.

87. Chang Y, Zhou C, Fan L, Qiu G, Wang G, Wei G, Chang X and Li X: Upregulation of microRNA-300 induces the proliferation of liver cancer by downregulating transcription factor FOXO1. Oncol Rep 40: 3561-3572, 2018.

88. Xu H, Li G, Yue Z and Li C: HCV core protein-induced upregulation of microRNA-196a promotes aberrant proliferation in hepatocellular carcinoma by targeting FOXO1. Mol Med Rep 13: 5223-5229, 2016

89. Yang XW, Shen GZ, Cao LQ, Jiang XF, Peng HP, Shen G, Chen D and Xue P: MicroRNA-1269 promotes proliferation in human hepatocellular carcinoma via downregulation of FOXO1. BMC Cancer 14: 909, 2014

90. Xu X, Yamamoto H, Sakon M, Yasui M, Ngan CY, Fukunaga H, Morita T, Ogawa M, Nagano H, Nakamori S, et al: Overexpression of CDC25A phosphatase is associated with hypergrowth activity and poor prognosis of human hepatocellular carcinomas. Clin Cancer Res 9: 1764-1772, 2003.

91. Xu X, Yamamoto H, Liu G, Ito Y, Ngan CY, Kondo M, Nagano H, Dono K, Sekimoto M and Monden M: CDC25A inhibition suppresses the growth and invasion of human hepatocellular carcinoma cells. Int J Mol Med 21: 145-152, 2008.

92. Kelly P, Moeller BJ, Juneja J, Booden MA, Der CJ, Daaka Y, Dewhirst MW, Fields TA and Casey PJ: The G12 family of heterotrimeric $\mathrm{G}$ proteins promotes breast cancer invasion and metastasis. Proc Natl Acad Sci USA 103: 8173-8178, 2006.

93. Kelly P, Stemmle LN, Madden JF, Fields TA, Daaka Y and Casey PJ: A role for the G12 family of heterotrimeric G proteins in prostate cancer invasion. J Biol Chem 281: 26483-26490, 2006.

94. Chia CY, Kumari U and Casey PJ: Breast cancer cell invasion mediated by Ga12 signaling involves expression of interleukins- 6 and -8, and matrix metalloproteinase-2. J Mol Signal 9: 6, 2014.

95. Udayappan UK and Casey PJ: c-Jun contributes to transcriptional control of GNA12 expression in prostate cancer cells. Molecules 22, 2017.

96. Bakiri L, Hamacher R, Graña O, Guío-Carrión A, Campos-Olivas R, Martinez L, Dienes HP, Thomsen MK, Hasenfuss SC and Wagner EF: Liver carcinogenesis by FOS-dependent inflammation and cholesterol dysregulation. J Exp Med 214: 1387-1409, 2017.

97. Watanabe T, Hiasa Y, Tokumoto Y, Hirooka M, Abe M, Ikeda Y, Matsuura B, Chung RT and Onji M: Protein kinase R modulates c-Fos and c-Jun signaling to promote proliferation of hepatocellular carcinoma with hepatitis $\mathrm{C}$ virus infection. PLoS One 8: e67750, 2013.

98. Fan Q, He M, Deng X, Wu WK, Zhao L, Tang J, Wen G, Sun X and Liu Y: Derepression of c-Fos caused by microRNA-139 down-regulation contributes to the metastasis of human hepatocellular carcinoma. Cell Biochem Funct 31: 319-324, 2013.

(i) $(5)$ This work is licensed under a Creative Commons Attribution-NonCommercial-NoDerivatives 4.0 International (CC BY-NC-ND 4.0) License. 